\title{
ULTRADISCRETIZATION OF A SOLVABLE TWO-DIMENSIONAL CHAOTIC MAP ASSOCIATED WITH THE HESSE CUBIC CURVE
}

\author{
Kenji KAJIWARA, Masanobu KANEKO, Atsushi NOBE and Teruhisa TSUDA \\ (Received 3 March 2009)
}

\begin{abstract}
We present a solvable two-dimensional piecewise linear chaotic map that arises from the duplication map of a certain tropical cubic curve. Its general solution is constructed by means of the ultradiscrete theta function. We show that the map is derived by the ultradiscretization of the duplication map associated with the Hesse cubic curve. We also show that it is possible to obtain the non-trivial ultradiscrete limit of the solution in spite of a problem known as 'the minus-sign problem.'
\end{abstract}

\section{Introduction}

Ultradiscretization [41] has been widely recognized as a powerful tool to extend the theory of integrable systems to piecewise linear discrete dynamical systems (ultradiscrete systems) $[7,11,23,29,31,36,37,39,44,47,50]$. In particular, it yields various soliton cellular automata when it is possible to discretize the dependent variables into a finite number of integers by a suitable choice of parameters. It has also established the links between the theory of integrable systems and various areas of mathematical sciences, such as combinatorics, representation theory, tropical geometry, traffic flow models, and so on $[5,6,10,13,17,19,20,21,22,27,30,31,33,46,43,45,49]$. One of the key features of ultradiscretization is that one can obtain piecewise linear discrete dynamical systems from rational discrete dynamical systems by a certain limiting procedure, which corresponds to the low-temperature limit in statistical mechanics. When this procedure is applied to a certain class of discrete integrable systems, wide classes of exact solutions, such as soliton solutions or periodic solutions, survive under the limit, which yield exact solutions to the ultradiscrete integrable systems.

While the application of ultradiscretization to integrable systems has achieved great success, it seems that only a few results have been reported on the application to nonintegrable systems $[\mathbf{3 8}, \mathbf{4 0}]$. One reason may be that, in many cases of non-integrable systems, fundamental properties are lost under the limit. For example, it is possible to ultradiscretize the celebrated logistic map formally, but its chaotic behavior is lost through the ultradiscretization [16].

2000 Mathematics Subject Classification: Primary 14H40, 14H52, 14K25, 37E05, 37F10.

Keywords: ultradiscretization; tropical geometry; theta functions; plane cubic curve; discrete dynamical systems.

(C) 2009 Faculty of Mathematics, Kyushu University 
In [16], the ultradiscretization of a one-dimensional chaotic map that arises as the duplication formula of Jacobi's sn function has been considered. It exemplifies a solvable chaotic system, which is regarded as a dynamical system lying on the border of integrability and chaos [4], in the sense that, though its exact solution is given by an elliptic function, its dynamics exhibits typical chaotic behaviors such as irreversibility, sensitivity to initial values, positive entropy, and so on. By applying the ultradiscretization, it has been shown that we obtain the tent map and its general solution simultaneously. Moreover, a tropical geometric interpretation of the tent map has been presented; namely, it arises as the duplication map on a certain tropical biquadratic curve. This result implies that there is the world of elliptic curves and elliptic functions behind the tent map, which might be an unexpected and interesting viewpoint. It also suggests that the tropical geometry and ultradiscretization provide a theoretical framework for the description of such a geometric aspect.

In this paper, we present two kinds of two-dimensional solvable chaotic maps and their general solutions that are directly connected through the ultradiscretization. In Section 2, we construct a piecewise linear map from a duplication map on a certain tropical plane cubic curve. We also construct its general solution in terms of the ultradiscrete theta function $[16,20,26,32,33,42]$ by using the tropical Abel-Jacobi map. In Section 3, we consider a certain rational map that arises as a duplication map on the Hesse cubic curve (see, for example, $[\mathbf{1}, \mathbf{1 5}, \mathbf{3 5}]$ ), whose general solution is expressible in terms of theta functions of level three. In Section 4, we discuss the ultradiscretization of the rational map and its solution obtained in Section 3, and show that they yield the piecewise linear map and its solution obtained in Section 2. The rational map and its general solutions discussed in Sections 3 and 4 involve a problem known as 'the minus-sign problem,' which is usually regarded as an obstacle to successful application of ultradiscretization. We show that it is possible to overcome the problem by taking careful parametrization and a limiting procedure.

\section{Duplication map on tropical cubic curve}

\subsection{Duplication map}

In this section, we construct the duplication map on a certain tropical curve. For the basic notions of tropical geometry, we refer to $[3, \mathbf{1 2}, \mathbf{2 4 - 2 6 , 3 4 ]}$.

Let us consider the tropical curve $C_{K}$ given by the tropical polynomial

$$
\Psi(X, Y ; K)=\max [3 X, 3 Y, X+Y+K, 0], \quad X, Y, K \in \mathbb{R}, K>0 .
$$

The curve $C_{K}$ is defined as the set of points where $\Psi$ is not differentiable. As shown in Figure 1(a), the vertices $V_{i}$ and the edges $E_{i}$ of $C_{K}$ are given by $V_{1}=(-K, 0)$, $V_{2}=(0,-K), V_{3}=(K, K)$ and $E_{1}=V_{1} V_{2}, E_{2}=V_{2} V_{3}, E_{3}=V_{3} V_{1}$, respectively. From the Newton subdivision of the support of $C_{K}$ given in Figure 1(b), we see that $C_{K}$ is a degreethree curve. For a vertex on the tropical curve, let $\boldsymbol{v}_{i} \in \mathbb{Z}^{2}(i=1, \ldots, n)$ be the primitive tangent vectors along the edges emanating from the vertex. Then it is known that for any vertex there exist natural numbers $w_{i} \in \mathbb{Z}_{>0}(i=1, \ldots, n)$ such that the following balancing condition holds:

$$
w_{1} \boldsymbol{v}_{1}+\cdots+w_{n} \boldsymbol{v}_{n}=(0,0)
$$




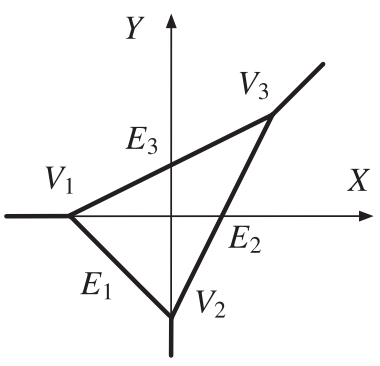

(a)

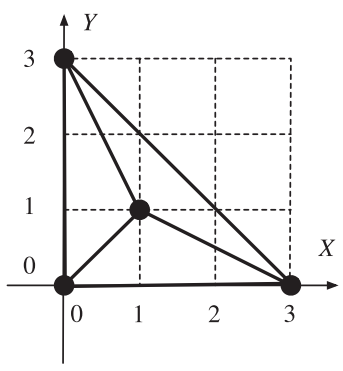

(b)

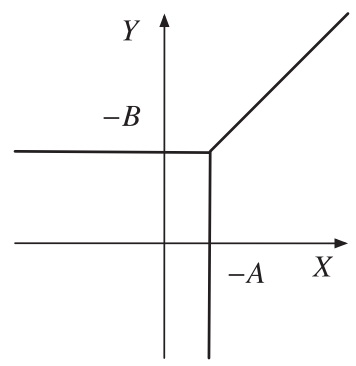

(c)

Figure 1. (a) Tropical curve $C_{K} ; V_{1}=(-K, 0), V_{2}=(0,-K), V_{3}=(K, K)$. Primitive tangent vector of each edge: $E_{1}, \boldsymbol{v}_{1}=(1,-1) ; E_{2}, \boldsymbol{v}_{2}=(1,2) ; E_{3}, \boldsymbol{v}_{3}=(2,1)$. (b) Newton subdivision of the support of $C_{K}$. (c) Tropical line.

We call $w_{i}$ the weight of the corresponding edge. Now, since the primitive tangent vectors emanating from $V_{1}$ are given by $(-1,0),(1,-1)$ and $(2,1)$, the balancing condition at $V_{1}$ is given by

$$
3(-1,0)+(1,-1)+(2,1)=(0,0) .
$$

Therefore, the weights of $E_{1}, E_{3}$ and the tentacle along the edges emanating from $V_{1}$ are given by 1,1 and 3 , respectively. The balancing conditions at $V_{2}$ and $V_{3}$ show that the weights of the edges $E_{i}(i=1,2,3)$ are 1 , and those of the tentacles of $C_{K}$ are all 3, respectively. If a vertex $V$ is three-valent, namely $V$ has exactly three adjacent edges whose primitive tangent vectors and weights are $\boldsymbol{v}_{i}$ and $w_{i}(i=1,2,3)$, respectively, the multiplicity of $V$ is defined by $w_{1} w_{2}\left|\operatorname{det}\left(\boldsymbol{v}_{1}, \boldsymbol{v}_{2}\right)\right|=w_{2} w_{3}\left|\operatorname{det}\left(\boldsymbol{v}_{2}, \boldsymbol{v}_{3}\right)\right|=w_{3} w_{1}\left|\operatorname{det}\left(\boldsymbol{v}_{3}, \boldsymbol{v}_{1}\right)\right|$. If all the vertices of the tropical curve are three-valent and have multiplicity one, then the curve is said to be smooth. The multiplicity of the vertex $V_{1}$ is computed as

$$
3 \times 1 \times\left|\operatorname{det}\left(\begin{array}{rr}
-1 & 0 \\
1 & -1
\end{array}\right)\right|=3,
$$

and similarly those of $V_{2}$ and $V_{3}$ are both three, which imply that $C_{K}$ is not smooth. The genus is equal to the first Betti number of $C_{K}$, which is one as shown in Figure 1(a). Thus the curve $C_{K}$ is a non-smooth, degree-three tropical curve of genus one. Note that the cycle $\bar{C}_{K}$ of $C_{K}$ (the triangle obtained by removing the tentacles from $C_{K}$ ) can be given by the equation

$$
\max [3 X, 3 Y, 0]=X+Y+K .
$$

A tropical line is the tropical curve given by the tropical polynomial of the form

$$
L(X, Y)=\max [X+A, Y+B, 0],
$$

which is shown in Figure 1(c). The three primitive tangent vectors emanating from the vertex are given by $(-1,0),(0,-1)$ and $(1,1)$. From the balancing condition $(-1,0)+(0,-1)+$ $(1,1)=(0,0)$, the weights of the edges are all 1 .

Vigeland [48] has introduced the group law on the tropical elliptic curve, which is a smooth, degree-three curve of genus one. According to the group law, the duplication 
map is formulated as follows. Let $C$ be a tropical elliptic curve and let $\bar{C}$ be its cycle. Take a point $P \in \bar{C}$. We draw a tropical line that intersects with $\bar{C}$ at $P$ with the intersection multiplicity two, and denote the other intersection point by $P * P$. Drawing a tropical line passing through $\mathcal{O}$ and $P * P$ with a suitable choice of the origin of addition $\mathcal{O} \in \bar{C}$, the third intersection point is $2 P$.

For a given point $P$ on a tropical elliptic curve, the tropical line that intersects at $P$ with the intersection multiplicity two does not exist in general. However, the curve $C_{K}$ has a remarkable property that it is possible to draw a tropical line that intersects at any point on $\bar{C}_{K}$ with the intersection multiplicity two. The explicit form of the duplication map is given as follows.

Proposition 2.1. Choosing the origin as $\mathcal{O}=V_{3}$, the duplication map $\bar{C}_{K} \ni P=$ $(X, Y) \longmapsto 2 P=(\bar{X}, \bar{Y}) \in \bar{C}_{K}$ on the tropical cubic curve $C_{K}$ is given by

$$
\bar{X}=Y+3 \max [0, X]-3 \max [X, Y], \quad \bar{Y}=X+3 \max [0, Y]-3 \max [X, Y],
$$

or

$$
\begin{aligned}
& X_{n+1}=Y_{n}+3 \max \left[0, X_{n}\right]-3 \max \left[X_{n}, Y_{n}\right], \\
& Y_{n+1}=X_{n}+3 \max \left[0, Y_{n}\right]-3 \max \left[X_{n}, Y_{n}\right],
\end{aligned}
$$

where $\left(X_{n}, Y_{n}\right)$ is the point obtained by the $n$ times successive applications of the map to $(X, Y)$.

Proof. Case 1: $P \in E_{1}$. As illustrated in Figure 2(a), the primitive tangent vectors of the two edges passing through $P$ are $(1,-1)$ (thick line) and $(1,1)$ (broken line), respectively, and the weights of the edges crossing at $P$ are both 1 . Then the intersection multiplicity is given by

$$
1 \times 1 \times\left|\operatorname{det}\left(\begin{array}{rr}
1 & -1 \\
1 & 1
\end{array}\right)\right|=2 .
$$

Let $P * P$ be the other intersection point. Note that the intersection multiplicity at $P * P$ is one. Then the map $P=(X, Y) \longmapsto P * P=\left(X^{\prime}, Y^{\prime}\right)$ is constructed as follows. Since $P \in E_{1}$ and $P * P \in E_{1} \cup E_{2}$, we have

$$
0=X+Y+K, \quad \max \left[3 X^{\prime}, 3 Y^{\prime}\right]=X^{\prime}+Y^{\prime}+K .
$$

Subtracting the second equation from the first one, we obtain, by using $\left(X-X^{\prime}\right) /\left(Y-Y^{\prime}\right)=$ 1 ,

$$
X^{\prime}=X-3 \max [X, Y], \quad Y^{\prime}=Y-3 \max [X, Y] .
$$

Our choice of the origin of addition $\mathcal{O}=V_{3}$ makes the form of $2 P$ simple. It is obvious, as illustrated in Figure $2(\mathrm{~b})$, that $2 P=(\bar{X}, \bar{Y})$ is given by $(\bar{X}, \bar{Y})=\left(Y^{\prime}, X^{\prime}\right)$. Hence we obtain the map $P \longmapsto 2 P$ as

$$
\bar{X}=Y-3 \max [X, Y], \quad \bar{Y}=X-3 \max [X, Y], \quad(X, Y) \in E_{1} .
$$

Case 2: $P \in E_{2}$. As illustrated in Figure 3(a), the two primitive tangent vectors of the edges passing through $P$ are $(1,2)$ and $(1,0)$, and the weights of the edges crossing at $P$ are both 1 . Then the intersection multiplicity is given by

$$
1 \times 1 \times\left|\operatorname{det}\left(\begin{array}{cc}
1 & 2 \\
1 & 0
\end{array}\right)\right|=2 .
$$




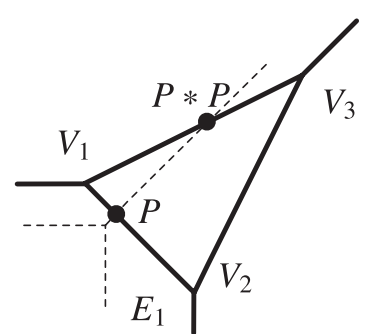

(a)

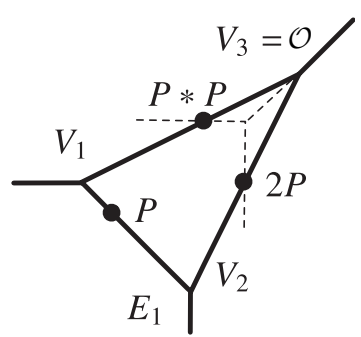

(b)

FIgure 2. (a) Map $P \mapsto P * P$ for $P \in E_{1}$. The intersection point of $C_{K}$ and the line passing through $P$ with multiplicity two (broken line) is $P * P$. (b) Map $P * P \mapsto 2 P$. The intersection point of $C_{K}$ and the line passing through $\mathcal{O}=V_{3}$ and $P * P$ (broken line) is $2 P$. Obviously $P * P$ and $2 P$ are symmetric with respect to $X=Y$.

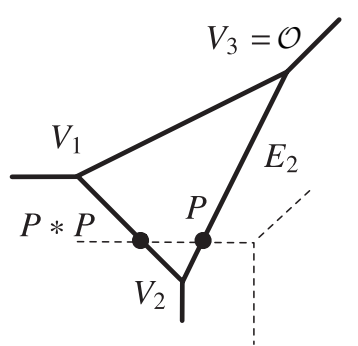

(a)

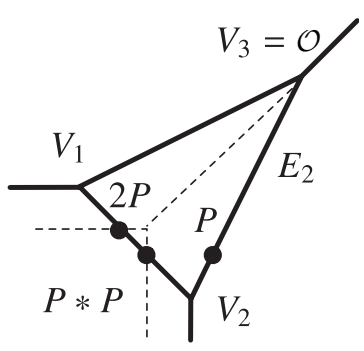

(b)

Figure 3. (a) Map $P \mapsto P * P$ for $P \in E_{2}$. (b) Map $P * P \mapsto 2 P$.

Since $P \in E_{2}$ and $P * P \in E_{1} \cup E_{3}$, we have

$$
3 X=X+Y+K, \quad \max \left[3 Y^{\prime}, 0\right]=X^{\prime}+Y^{\prime}+K .
$$

By using $Y^{\prime}=Y$, we obtain the map $P \longmapsto P * P$ and $P \longmapsto 2 P$ as

$$
\begin{gathered}
X^{\prime}=-2 X+3 \max [0, Y], \quad Y^{\prime}=Y, \\
\bar{X}=Y, \quad \bar{Y}=-2 X+3 \max [0, Y], \quad(X, Y) \in E_{2},
\end{gathered}
$$

respectively.

Case 3: $P \in E_{3}$. As illustrated in Figure 4(a), the two primitive tangent vectors of the edges passing through $P$ are $(2,1)$ and $(0,1)$, and the weights of the edges crossing at $P$ are both 1 . The intersection multiplicity is given by

$$
1 \times 1 \times\left|\operatorname{det}\left(\begin{array}{ll}
2 & 1 \\
0 & 1
\end{array}\right)\right|=2 .
$$

Since $P \in E_{3}$ and $P * P \in E_{1} \cup E_{2}$, we have

$$
3 Y=X+Y+K, \quad \max \left[3 X^{\prime}, 0\right]=X^{\prime}+Y^{\prime}+K .
$$




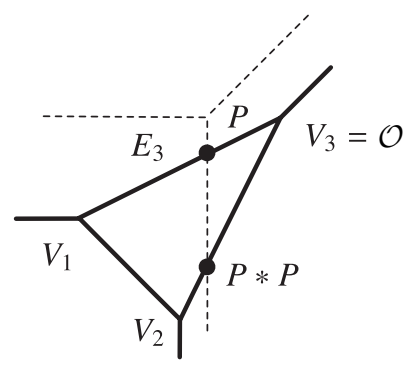

(a)

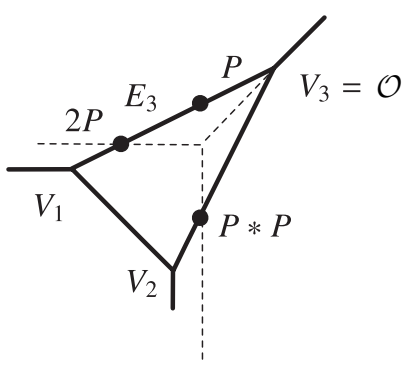

(b)

Figure 4. (a) Map $P \mapsto P * P$ for $P \in E_{3}$. (b) Map $P * P \mapsto 2 P$.

By using $X^{\prime}=X$, we obtain the map $P \mapsto P * P$ and $P \mapsto 2 P$ as

$$
\begin{gathered}
X^{\prime}=X, \quad Y^{\prime}=-2 Y+3 \max [0, X], \\
\bar{X}=-2 Y+3 \max [0, X], \quad \bar{Y}=X, \quad(X, Y) \in E_{3},
\end{gathered}
$$

respectively.

We finally obtain equation (2.7) by collecting equations (2.12), (2.16) and (2.20) together.

Strictly speaking, the group law in [48] cannot be applied to our case, since $C_{K}$ is not a smooth curve. However, it is possible to show by direct computation that the map (2.8) is actually a duplication map on the tropical Jacobian of $\bar{C}_{K}$. For this purpose, we first compute the total lattice length $\mathcal{L}$ of $\bar{C}_{K}$, which is defined by the sum of the length of each edge scaled by the norm of corresponding primitive tangent vector:

$$
\mathcal{L}=\sum_{i=1}^{3} \frac{\left|E_{i}\right|}{\left|\boldsymbol{v}_{i}\right|}=\frac{\sqrt{5} K}{\sqrt{5}}+\frac{\sqrt{2} K}{\sqrt{2}}+\frac{\sqrt{5} K}{\sqrt{5}}=3 K .
$$

Then the tropical Jacobian $J\left(\bar{C}_{K}\right)$ of $\bar{C}_{K}$ is given by

$$
J\left(\bar{C}_{K}\right)=\mathbb{R} / \mathcal{L} \mathbb{Z}=\mathbb{R} / 3 K \mathbb{Z} .
$$

The Abel-Jacobi map $\eta: \bar{C}_{K} \rightarrow J\left(\bar{C}_{K}\right)$ is defined as the piecewise linear map satisfying

$$
\eta(\mathcal{O})=\eta\left(V_{3}\right)=0, \quad \eta\left(V_{1}\right)=\frac{\left|E_{3}\right|}{\left|\boldsymbol{v}_{3}\right|}=K, \quad \eta\left(V_{2}\right)=\eta\left(V_{1}\right)+\frac{\left|E_{2}\right|}{\left|\boldsymbol{v}_{2}\right|}=2 K
$$

Proposition 2.2. The map $\bar{C}_{K} \ni P=(X, Y) \longmapsto \bar{P}=(\bar{X}, \bar{Y}) \in \bar{C}_{K}$ defined by equation (2.7) is a duplication map on the Jacobian $J\left(\bar{C}_{K}\right)$. Namely, we have $\eta(\bar{P})=$ $2 \eta(P) \bmod 3 K$.

Proof. We consider the case $P \in E_{1}$. Suppose $P=(X, Y)$ satisfies $V_{1} P: V_{2} P=s: 1-s$ $(0 \leq s \leq 1)$, namely

$$
P=(X, Y)=(-(1-s) K,-s K), \quad \eta(P)=\eta\left(V_{1}\right)+s K=(1+s) K .
$$




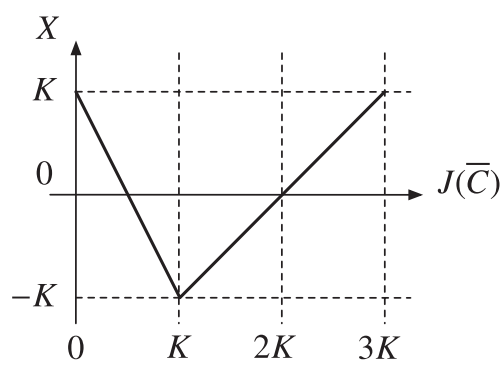

(a)

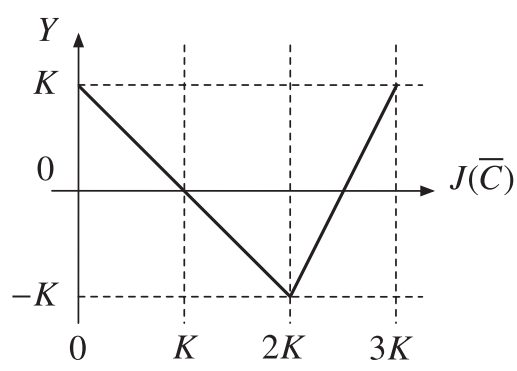

(b)

Figure 5. (a) $\pi_{1} \circ \eta^{-1}: J\left(\bar{C}_{K}\right) \rightarrow X$. (b) $\pi_{2} \circ \eta^{-1}: J\left(\bar{C}_{K}\right) \rightarrow Y$.

Case (I): $X \leq Y\left(0 \leq s \leq \frac{1}{2}\right)$. From equation (2.12), $\bar{P}$ is given by

$$
\bar{X}=Y-3 Y=-2 Y=2 s K, \quad \bar{Y}=X-3 Y=(-1+4 s) K, \quad \bar{P} \in E_{2},
$$

which implies that

$$
V_{2} \bar{P}: V_{3} \bar{P}=2 s: 1-2 s, \quad \eta(\bar{P})=\eta\left(V_{2}\right)+2 s K=2(1+s) K=2 \eta(P) .
$$

Case (II): $X \geq Y\left(\frac{1}{2} \leq s \leq 1\right)$. In this case, $\bar{P}$ is given by

$$
\bar{X}=Y-3 X=(3-4 s) K, \quad \bar{Y}=-2 X=2(1-s) K, \quad \bar{P} \in E_{3},
$$

which implies that

$$
V_{3} \bar{P}: V_{1} \bar{P}=-1+2 s: 2(1-s), \quad \eta(\bar{P})=(-1+2 s) K \equiv 2(1+s) K=2 \eta(P) \bmod 3 K .
$$

Therefore we have shown that $\eta(\bar{P})=2 \eta(P)$ for $P \in E_{1}$. We omit the proof of other cases since they can be shown in a similar manner.

\subsection{General solution}

From the construction of the map (2.8), it is possible to obtain the general solution by using the Abel-Jacobi map of $\bar{C}_{K}$. Let $\pi_{1}$ and $\pi_{2}$ be projections from $\bar{C}_{K}$ to the $X$-axis and the $Y$-axis, respectively. Then the maps $\pi_{1} \circ \eta^{-1}$ and $\pi_{2} \circ \eta^{-1}$, namely, the maps from the tropical Jacobian to the $X$-axis and the $Y$-axis through the Abel-Jacobi map, are given as illustrated in Figures 5(a) and (b), respectively. Therefore, Proposition 2.2 implies that $X_{n}=\pi_{1} \circ \eta^{-1}\left(2^{n} u_{0}\right)$ and $Y_{n}=\pi_{2} \circ \eta^{-1}\left(2^{n} u_{0}\right)$ for arbitrary $u_{0} \in J\left(\bar{C}_{K}\right)$ gives the general solution to equation (2.8).

It is possible to express $\pi_{1} \circ \eta^{-1}$ and $\pi_{2} \circ \eta^{-1}$ by using the ultradiscrete theta function $\Theta(u ; \theta)$ defined by $[\mathbf{1 6}, \mathbf{2 0}, \mathbf{2 6}, \mathbf{3 2}, \mathbf{3 3}, \mathbf{4 2}]$

$$
\Theta(u ; \theta)=-\theta\left\{((u))-\frac{1}{2}\right\}^{2}, \quad((u))=u-\text { Floor }(u) .
$$

For this purpose, we introduce a piecewise linear periodic function $S(u ; \alpha, \beta, \theta)$ by

$$
S(u ; \alpha, \beta, \theta)=\Theta\left(\frac{u}{\alpha} ; \theta\right)-\Theta\left(\frac{u-\beta}{\alpha} ; \theta\right),
$$




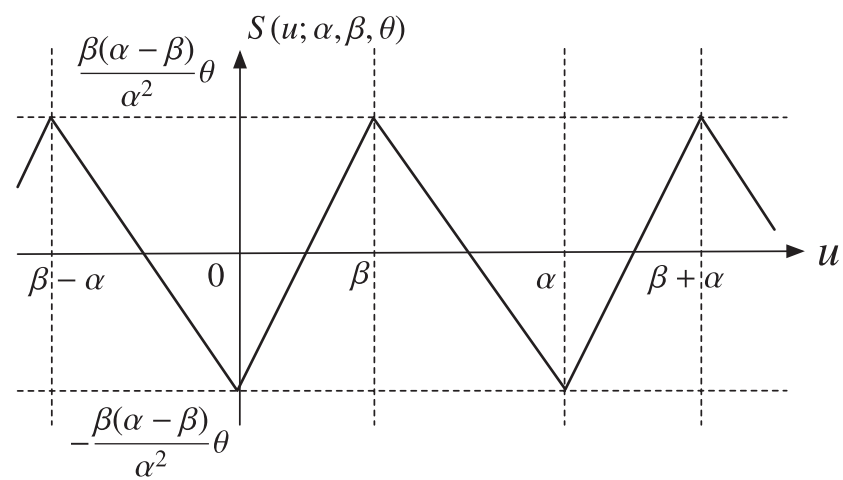

FIGURE 6. Graph of $S(u ; \alpha, \beta, \theta)$.

which has a period $\alpha$ and amplitude $2 \beta(\alpha-\beta) \theta / \alpha^{2}$ as illustrated in Figure 6. Comparing Figure 5 with Figure 6, we have

$$
\pi_{1} \circ \eta^{-1}(u)=S\left(u-K ; 3 K, 2 K, \frac{9}{2} K\right), \quad \pi_{2} \circ \eta^{-1}(u)=S\left(u-2 K ; 3 K, K, \frac{9}{2} K\right) .
$$

Therefore, we obtain the following proposition.

Proposition 2.3. For a given initial value $P_{0}=\left(X_{0}, Y_{0}\right)$, the general solution to the map (2.8) is given by

$$
\begin{aligned}
X_{n} & =S\left(2^{n} u_{0}-K ; 3 K, 2 K, \frac{9}{2} K\right), & Y_{n} & =S\left(2^{n} u_{0}-2 K ; 3 K, K, \frac{9}{2} K\right), \\
K & =3 \max \left[X_{0}, Y_{0}, 0\right]-X_{0}-Y_{0}, & u_{0} & =\eta\left(P_{0}\right) .
\end{aligned}
$$

Figure 7 shows the orbit of the map (2.8) plotted with 3000 times iterations. The map has an invariant curve $\bar{C}_{K}$ given by equation (2.5), and the figure shows that the curve is filled with the points of the orbit.

\section{Duplication map on the Hesse cubic curve}

\subsection{Duplication map}

The Hesse cubic curve is a curve in $\mathbb{P}^{2}$ given by

$$
E_{\mu}: \quad x^{3}+y^{3}+1=3 \mu x y,
$$

or in the homogeneous coordinates $\left[x_{0}: x_{1}: x_{2}\right]=[x: y: 1]$ by

$$
E_{\mu}: \quad x_{0}^{3}+x_{1}^{3}+x_{2}^{3}=3 \mu x_{0} x_{1} x_{2} .
$$

The nine inflection points are given by $[1:-1: 0],[1:-\omega: 0],\left[1:-\omega^{2}: 0\right],[1: 0:-1]$, $[1: 0:-\omega],\left[1: 0:-\omega^{2}\right],[0: 1:-1],[0: 1:-\omega]$ and $\left[0: 1:-\omega^{2}\right]$, where $\omega$ is a non-trivial third root of 1 . It is known that any non-singular plane cubic curve is projectively equivalent to $E_{\mu}$ (see e.g. [1]). Moreover, these inflection points are also the base points of the pencil

$$
t_{0}\left(x_{0}^{3}+x_{1}^{3}+x_{2}^{3}\right)=t_{1} x_{0} x_{1} x_{2}, \quad\left[t_{0}: t_{1}\right] \in \mathbb{P}^{1} .
$$




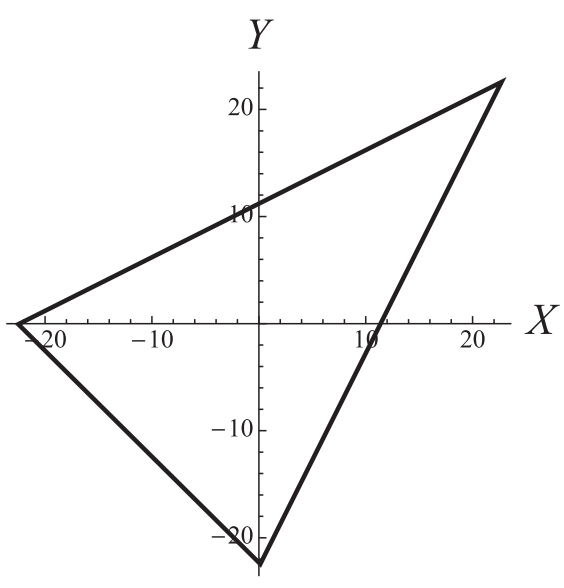

(a)

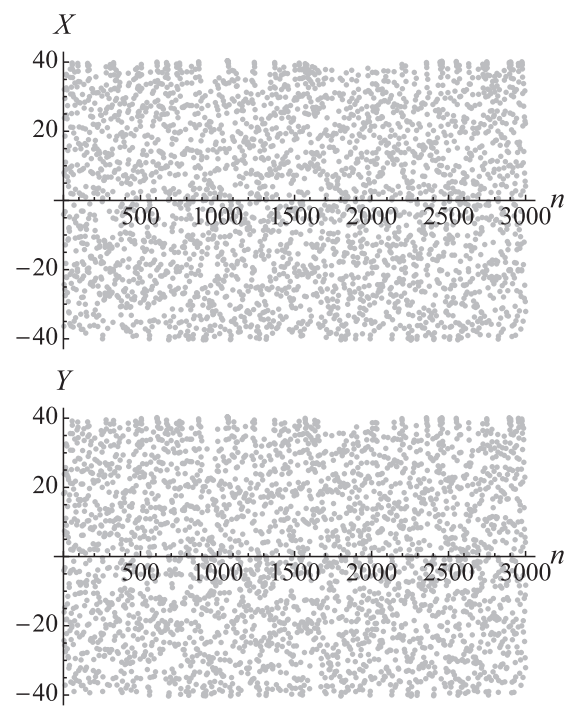

(b)

FIGURE 7. Orbit of the map (2.8) with the initial value $\left(X_{0}, Y_{0}\right)=(8.56546,15.6231)$.

The duplication map is constructed by the standard procedure. For an arbitrary point on $P \in E_{\mu}$, draw a tangent line, and set the other intersection of the tangent line and $E_{\mu}$ as $P * P$. Taking one of the inflection points as an origin $\mathcal{O}$ of addition, the intersection of $E_{\mu}$ and the line connecting $P * P$ and $\mathcal{O}$ gives $2 P$. Choosing $\mathcal{O}$ to be $[1:-1: 0]$ among the nine inflection points of $E_{\mu}$, the duplication map $P=(x, y) \mapsto 2 P=(\bar{x}, \bar{y})$ is explicitly calculated as (see, for example, $[\mathbf{1 5}, \mathbf{3 5}]$ )

$$
\bar{x}=\frac{\left(1-x^{3}\right) y}{x^{3}-y^{3}}, \quad \bar{y}=\frac{\left(1-y^{3}\right) x}{y^{3}-x^{3}},
$$

or writing the point obtained by the $n$ times applications of the map to $(x, y)$ as $\left(x_{n}, y_{n}\right)$, we have

$$
x_{n+1}=\frac{\left(1-x_{n}^{3}\right) y_{n}}{x_{n}^{3}-y_{n}^{3}}, \quad y_{n+1}=\frac{\left(1-y_{n}^{3}\right) x_{n}}{y_{n}^{3}-x_{n}^{3}} .
$$

By construction, it is obvious that the map (3.5) has the invariant curve $E_{\mu}$, where $\mu$ is the conserved quantity. Figure 8 shows the orbit of the map (3.5) plotted with 3000 times iterations. Note that, although the invariant curve has a component in the first quadrant $x, y>0$ for $\mu>0$, the real orbit never enters this quadrant (except for the initial point), which can be verified by a simple consideration. Suppose that $x_{n}>0$ at some $n$. Then equation (3.5) implies that $\left(x_{n-1}, y_{n-1}\right)$ must be in the highlighted region of Figure 9(a). On the other hand, if $y_{n}>0$ at some $n,\left(x_{n-1}, y_{n-1}\right)$ must be in the highlighted region of Figure 9(b). Since the intersection of the two regions is empty, it is impossible to realize $x_{n}, y_{n}>0$ for any $n$ as long as we start from the real initial value. 


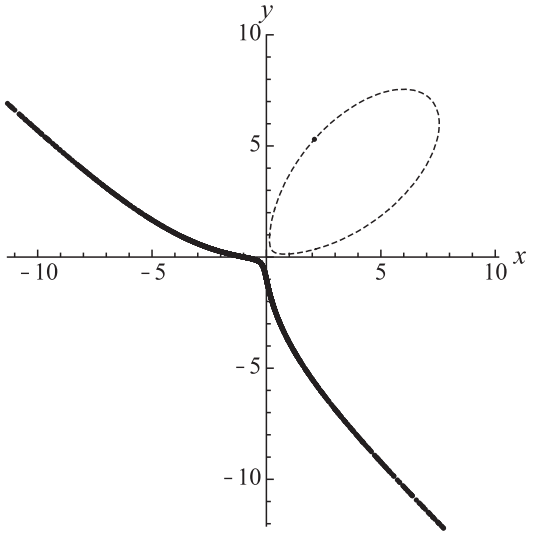

(a)

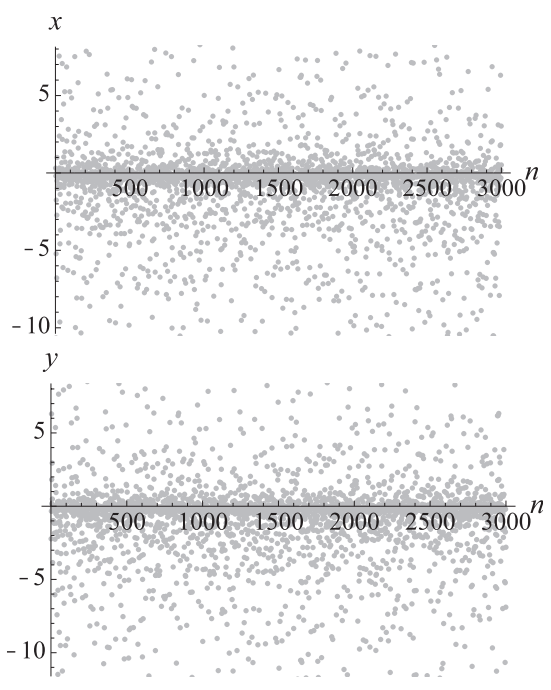

(b)

FIGURE 8. Orbit of the map (3.5) with the initial value $\left(x_{0}, y_{0}\right)=(2.1,5.3)$. The dashed line in (a) is the invariant curve.

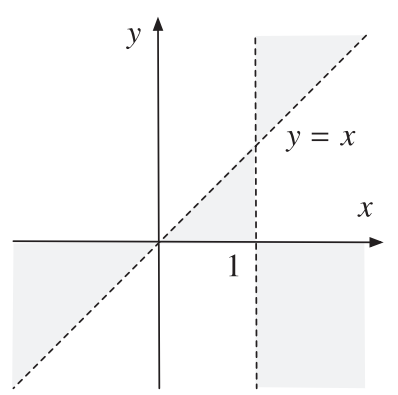

(a)

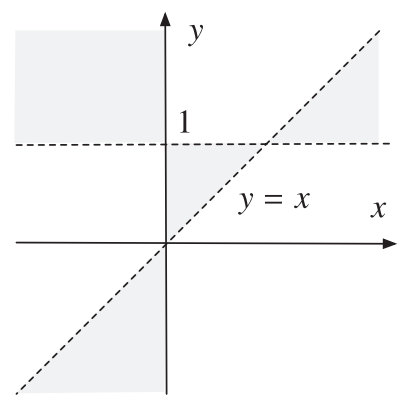

(b)

FIGURE 9. (a) Region of $\left(x_{n-1}, y_{n-1}\right)$ for $x_{n}>0$. (b) Region of $\left(x_{n-1}, y_{n-1}\right)$ for $y_{n}>0$.

\subsection{General solution}

The general solution to the map (3.4) or (3.5) is given in terms of the following theta functions of level three. Let us introduce the functions $\theta_{k}(z, \tau)(k=0,1,2)$ by

$$
\theta_{k}(z, \tau)=\sum_{n \in \mathbb{Z}} e^{3 \pi i(n+k / 3-1 / 6)^{2} \tau} e^{6 \pi i(n+k / 3-1 / 6)(z+1 / 2)}=\vartheta_{(k / 3-1 / 6,3 / 2)}(3 z, 3 \tau),
$$

where $\vartheta_{(a, b)}(z, \tau)$ is the theta function with characteristic $(a, b)$ defined by

$$
\vartheta_{(a, b)}(z, \tau)=\sum_{n \in \mathbb{Z}} e^{\pi i(n+a)^{2} \tau+2 \pi i(n+a)(z+b)}, \quad \tau \in \mathcal{H}=\{\operatorname{Im} z>0, z \in \mathbb{C}\} .
$$


PROPOSITION 3.1. The general solution to equation (3.5) is given by

$$
x_{n}=\frac{\theta_{0}\left(2^{n} z_{0}, \tau\right)}{\theta_{2}\left(2^{n} z_{0}, \tau\right)}, \quad y_{n}=\frac{\theta_{1}\left(2^{n} z_{0}, \tau\right)}{\theta_{2}\left(2^{n} z_{0}, \tau\right)},
$$

where $z_{0} \in \mathbb{C}$ is an arbitrary constant.

Proposition 3.1 is a direct consequence of the following proposition.

\section{PROPOSITION 3.2.}

(1) $\theta_{k}(z, \tau)(k=0,1,2)$ satisfy

$$
\begin{gathered}
\theta_{0}(z, \tau)^{3}+\theta_{1}(z, \tau)^{3}+\theta_{2}(z, \tau)^{3}=3 \mu(\tau) \theta_{0}(z, \tau) \theta_{1}(z, \tau) \theta_{2}(z, \tau), \\
\mu(\tau)=-\frac{\varphi^{\prime}(0, \tau)}{\psi^{\prime}(0, \tau)},
\end{gathered}
$$

where

$$
\varphi(z, \tau)=\frac{\theta_{1}(z, \tau)}{\theta_{0}(z, \tau)}, \quad \psi(z, \tau)=\frac{\theta_{2}(z, \tau)}{\theta_{0}(z, \tau)} .
$$

(2) $\theta_{k}(z, \tau)(k=0,1,2)$ satisfy the following duplication formulas:

$$
\begin{aligned}
& \theta_{0}(0, \tau)^{3} \theta_{0}(2 z, \tau)=\theta_{1}(z, \tau)\left[\theta_{2}(z, \tau)^{3}-\theta_{0}(z, \tau)^{3}\right], \\
& \theta_{0}(0, \tau)^{3} \theta_{1}(2 z, \tau)=\theta_{0}(z, \tau)\left[\theta_{1}(z, \tau)^{3}-\theta_{2}(z, \tau)^{3}\right], \\
& \theta_{0}(0, \tau)^{3} \theta_{2}(2 z, \tau)=\theta_{2}(z, \tau)\left[\theta_{0}(z, \tau)^{3}-\theta_{1}(z, \tau)^{3}\right] .
\end{aligned}
$$

It seems that the above formulas are well known [2], but it might be useful for nonexperts to give an elementary proof here. In the following, we fix $\tau \in \mathcal{H}$ and write $\theta_{k}(z, \tau)=$ $\theta_{k}(z)$.

LEMMA 3.3. $\theta_{k}(z, \tau)(k=0,1,2)$ satisfy the following addition formulas:

$$
\begin{aligned}
& \theta_{0}(0)^{2} \theta_{0}(x+y) \theta_{0}(x-y)=\theta_{1}(x) \theta_{2}(x) \theta_{2}(y)^{2}-\theta_{0}(x)^{2} \theta_{0}(y) \theta_{1}(y), \\
& \theta_{0}(0)^{2} \theta_{1}(x+y) \theta_{0}(x-y)=\theta_{0}(x) \theta_{1}(x) \theta_{1}(y)^{2}-\theta_{2}(x)^{2} \theta_{0}(y) \theta_{2}(y), \\
& \theta_{0}(0)^{2} \theta_{2}(x+y) \theta_{0}(x-y)=\theta_{0}(x) \theta_{2}(x) \theta_{0}(y)^{2}-\theta_{1}(x)^{2} \theta_{1}(y) \theta_{2}(y), \\
& \theta_{0}(0)^{2} \theta_{0}(x+y) \theta_{1}(x-y)=\theta_{0}(x) \theta_{1}(x) \theta_{0}(y)^{2}-\theta_{2}(x)^{2} \theta_{1}(y) \theta_{2}(y), \\
& \theta_{0}(0)^{2} \theta_{1}(x+y) \theta_{1}(x-y)=\theta_{0}(x) \theta_{2}(x) \theta_{2}(y)^{2}-\theta_{1}(x)^{2} \theta_{0}(y) \theta_{1}(y), \\
& \theta_{0}(0)^{2} \theta_{2}(x+y) \theta_{1}(x-y)=\theta_{1}(x) \theta_{2}(x) \theta_{1}(y)^{2}-\theta_{0}(x)^{2} \theta_{0}(y) \theta_{2}(y), \\
& \theta_{0}(0)^{2} \theta_{0}(x+y) \theta_{2}(x-y)=\theta_{0}(x) \theta_{2}(x) \theta_{1}(y)^{2}-\theta_{1}(x)^{2} \theta_{0}(y) \theta_{2}(y), \\
& \theta_{0}(0)^{2} \theta_{1}(x+y) \theta_{2}(x-y)=\theta_{1}(x) \theta_{2}(x) \theta_{0}(y)^{2}-\theta_{0}(x)^{2} \theta_{1}(y) \theta_{2}(y), \\
& \theta_{0}(0)^{2} \theta_{2}(x+y) \theta_{2}(x-y)=\theta_{0}(x) \theta_{1}(x) \theta_{2}(y)^{2}-\theta_{2}(x)^{2} \theta_{0}(y) \theta_{1}(y) .
\end{aligned}
$$

We give the proof of Lemma 3.3 in the appendix.

Proof of Proposition 3.2. The duplication formulas (3.11) are obtained by putting $x=y=z$ in equations (3.12), (3.13) and (3.14). In order to prove equations (3.9) and (3.10), we first 
note that it follows by definition that

$$
\theta_{0}(-z)=-\theta_{1}(z), \quad \theta_{2}(-z)=-\theta_{2}(z)
$$

and hence

$$
\theta_{1}(0)=-\theta_{0}(0), \quad \theta_{2}(0)=0
$$

From Lemma 3.3, we obtain the addition formulas for $\varphi(z)$ and $\psi(z)$ (see equation (3.10)) as

$$
\begin{aligned}
& \varphi(x+y)=\frac{\varphi(x) \varphi(y)^{2}-\psi(x)^{2} \psi(y)}{\varphi(x) \psi(x) \psi(y)^{2}-\varphi(y)}, \\
& \varphi(x+y)=\frac{\psi(x) \psi(y)^{2}-\varphi(x)^{2} \varphi(y)}{\varphi(x)-\psi(x)^{2} \varphi(y) \psi(y)}, \\
& \varphi(x+y)=\frac{\varphi(x) \psi(x)-\varphi(y) \psi(y)}{\psi(x) \varphi(y)^{2}-\varphi(x)^{2} \psi(y)},
\end{aligned}
$$

and

$$
\begin{aligned}
& \psi(x+y)=\frac{\psi(x)-\varphi(x)^{2} \varphi(y) \psi(y)}{\varphi(x) \psi(x) \psi(y)^{2}-\varphi(y)}, \\
& \psi(x+y)=\frac{\varphi(x) \psi(x) \varphi(y)^{2}-\psi(y)}{\varphi(x)-\psi(x)^{2} \varphi(y) \psi(y)}, \\
& \psi(x+y)=\frac{\varphi(x) \psi(y)^{2}-\psi(x)^{2} \varphi(y)}{\psi(x) \varphi(y)^{2}-\varphi(x)^{2} \psi(y)} .
\end{aligned}
$$

Differentiating equations (3.23) and (3.25) by $y$ and putting $y=0$, we have

$$
\varphi^{\prime}(x)=-\varphi^{\prime}(0) \varphi(x)-\psi^{\prime}(0) \psi(x)^{2}, \quad \varphi^{\prime}(x)=\frac{\psi^{\prime}(0)+2 \varphi^{\prime}(0) \varphi(x) \psi(x)+\psi^{\prime}(0) \varphi(x)^{3}}{\psi(x)},
$$

respectively. Here we have used

$$
\varphi(0)=-1, \quad \psi(0)=0,
$$

which follow from equation (3.22). Equating the right-hand sides of the two equations in (3.29), we have

$$
1+\varphi(x)^{3}+\psi(x)^{3}=-3 \frac{\varphi^{\prime}(0)}{\psi^{\prime}(0)} \varphi(x) \psi(x),
$$

which yields equation (3.9) by multiplying by $\theta_{0}(z)^{3}$. This completes the proof.

Consider the map

$$
\mathbb{C} \ni z \longmapsto\left[\theta_{0}(z): \theta_{1}(z): \theta_{2}(z)\right] \in \mathbb{P}^{2}(\mathbb{C}) .
$$

From the relations

$$
\theta_{k}(z+1)=-\theta_{k}(z), \quad \theta_{k}(z+\tau)=-e^{3 \pi i \tau-6 \pi i z} \theta_{k}(z) \quad(k=0,1,2),
$$

we see that this induces a map from the complex torus $L_{\tau}=\mathbb{C} /(\mathbb{Z}+\mathbb{Z} \tau)$ to $E_{\mu}$, which is known to give an isomorphism $L_{\tau} \simeq E_{\mu}$ (see e.g. [2]). Since $0 \mapsto[1:-1: 0]$, the addition 
formulas (3.12)-(3.20) induce the group structure on $E_{\tau}$ with the origin $[1:-1: 0]$. Denoting the addition of two points $\left[x_{0}: x_{1}: x_{2}\right]$ and $\left[x_{0}^{\prime}: x_{1}^{\prime}: x_{2}^{\prime}\right]$ as $\left[x_{0}: x_{1}: x_{2}\right] \oplus\left[x_{0}^{\prime}: x_{1}^{\prime}: x_{2}^{\prime}\right]$, equations (3.12)-(3.20) imply

$$
\begin{aligned}
& {\left[x_{0}: x_{1}: x_{2}\right] \oplus\left[x_{0}^{\prime}: x_{1}^{\prime}: x_{2}^{\prime}\right]} \\
& \quad=\left[x_{1} x_{2} x_{2}^{\prime 2}-x_{0}^{2} x_{0}^{\prime} x_{1}^{\prime}: x_{0} x_{1} x_{1}^{\prime 2}-x_{2}^{2} x_{0}^{\prime} x_{2}^{\prime}: x_{0} x_{2} x_{0}^{\prime 2}-x_{1}^{2} x_{1}^{\prime} x_{2}^{\prime}\right] \\
& \quad=\left[x_{0} x_{1} x_{0}^{\prime 2}-x_{2}^{2} x_{1}^{\prime} x_{2}^{\prime}: x_{0} x_{2} x_{2}^{\prime 2}-x_{1}^{2} x_{0}^{\prime} x_{1}^{\prime}: x_{1} x_{2} x_{1}^{\prime 2}-x_{0}^{2} x_{0}^{\prime} x_{2}^{\prime}\right] \\
& \quad=\left[x_{0} x_{2} x_{1}^{\prime 2}-x_{1}^{2} x_{0}^{\prime} x_{2}^{\prime}: x_{1} x_{2} x_{0}^{\prime 2}-x_{0}^{2} x_{1}^{\prime} x_{2}^{\prime}: x_{0} x_{1} x_{2}^{\prime 2}-x_{2}^{2} x_{0}^{\prime} x_{1}^{\prime}\right] .
\end{aligned}
$$

In particular, when the two points are equal, the duplication formula is given by

$$
2\left[x_{0}: x_{1}: x_{2}\right]=\left[x_{1}\left(x_{2}^{3}-x_{0}^{3}\right): x_{0}\left(x_{1}^{3}-x_{2}^{3}\right): x_{2}\left(x_{0}^{3}-x_{1}^{3}\right)\right] .
$$

Moreover, the inverse of $\left[x_{0}: x_{1}: x_{2}\right]$ is given by

$$
-\left[x_{0}: x_{1}: x_{2}\right]=\left[x_{1}: x_{0}: x_{2}\right] \text {. }
$$

We finally remark that $\mu$ can also be expressed as follows. Differentiating both equations in (3.10) and putting $z=0$, we have by using equation (3.22),

$$
\varphi^{\prime}(0)=2 \frac{\theta_{0}^{\prime}(0)}{\theta_{0}(0)}, \quad \psi^{\prime}(0)=\frac{\theta_{2}^{\prime}(0)}{\theta_{0}(0)},
$$

which yield

$$
\mu(\tau)=-\frac{\varphi^{\prime}(0)}{\psi^{\prime}(0)}=-2 \frac{\theta_{0}^{\prime}(0)}{\theta_{2}^{\prime}(0)} .
$$

\section{Ultradiscretization}

So far we have constructed the piecewise linear map (2.8) as the duplication map on the tropical cubic curve $C_{K}$, whose general solution is given by equation (2.32). We have also presented the rational map (3.5), which arises as the duplication map on the Hesse cubic curve $E_{\mu}$. The general solution of the map is given by equation (3.8). In this section, we establish a correspondence between the two maps and their general solutions by means of the ultradiscretization.

\subsection{Ultradiscretization of map}

The key to the ultradiscretization is the following formula:

$$
\lim _{\epsilon \rightarrow+0} \epsilon \log \left(e^{A / \epsilon}+e^{B / \epsilon}+\cdots\right)=\max [A, B, \ldots] .
$$

Putting

$$
x_{n}=e^{X_{n} / \epsilon}, \quad y_{n}=e^{Y_{n} / \epsilon}
$$

we have from equation (3.5)

$$
\begin{gathered}
X_{n+1}=\epsilon \log \left(1+e^{\left(3 X_{n}+\epsilon \pi i\right) / \epsilon}\right)+Y_{n}-\epsilon \log \left(e^{\left(3 X_{n} / \epsilon\right)}+e^{\left(3 Y_{n}+\epsilon \pi i\right) / \epsilon}\right), \\
Y_{n+1}=\epsilon \log \left(1+e^{\left(3 Y_{n}+\epsilon \pi i\right) / \epsilon}\right)+X_{n}-\epsilon \log \left(e^{\left(3 X_{n}\right) / \epsilon}+e^{\left(3 Y_{n}+\epsilon \pi i\right) / \epsilon}\right),
\end{gathered}
$$


which yield, in the limit $\epsilon \rightarrow+0$, equation (2.8):

$$
\begin{aligned}
X_{n+1} & =\max \left[0,3 X_{n}\right]+Y_{n}-\max \left[3 X_{n}, 3 Y_{n}\right], \\
Y_{n+1} & =\max \left[0,3 Y_{n}\right]+X_{n}-\max \left[3 X_{n}, 3 Y_{n}\right] .
\end{aligned}
$$

The limit of the invariant curve (3.1) yields $\bar{C}_{K}$ :

$$
\max [0,3 X, 3 Y]=X+Y+K,
$$

by the use of

$$
3 \mu(\tau)=e^{K / \epsilon} .
$$

In the above process of ultradiscretization, we have calculated formally, for example,

$$
\epsilon \log \left(1-e^{3 X_{n} / \epsilon}\right)=\epsilon \log \left(1+e^{\left(3 X_{n}+\epsilon \pi i\right) / \epsilon}\right) \longrightarrow \max [0,3 X] \quad(\epsilon \rightarrow+0) .
$$

However, when the original rational map contains minus signs, such formal calculation sometimes does not give a consistent result. This may happen, for example, when we consider the limit of the exact solutions simultaneously, or when we consider the limit of the maps that are representations of a certain group or algebra. In both cases, the cancellations caused by the minus signs play a crucial role on the level of rational maps, and the structure of the rational maps is lost because such cancellations do not happen after taking the limit. This problem is sometimes called the minus-sign problem.

Therefore, we usually consider the subtraction-free rational map to apply the ultradiscretization $[\mathbf{4 5}, \mathbf{4 6}, \mathbf{4 9}] \dagger$, or we try to transform the map to be subtraction-free if possible [16]. Unfortunately, it seems that the map (3.5) cannot be transformed to be subtraction-free by simple transformations. However, in this case, it is possible to obtain a valid ultradiscrete limit of the general solution in spite of the minus-sign problem.

Remark 4.1. The nine inflection points of the Hesse cubic curve correspond to the vertices of the tropical cubic curve $C_{K}$ in the following manner. Consider one of the inflection points $\left[x_{0}: x_{1}: x_{2}\right]=[1:-1: 0]=\left[e^{0 / \epsilon}: e^{(0+i \pi \epsilon) / \epsilon}: e^{-\infty / \epsilon}\right]$. Then putting $x_{i}=e^{X_{i} / \epsilon}(i=0,1,2)$ and taking the limit $\epsilon \rightarrow+0$, we have $\left[X_{0}: X_{1}: X_{2}\right]=[0: 0:-\infty]=[\infty: \infty: 0]$. Note here that on this level equivalence of the homogeneous coordinates is given by $\left[X_{0}: X_{1}: X_{2}\right]=$ $\left[X_{0}+L: X_{1}+L: X_{2}+L\right]$ for any constant $L$. In the inhomogeneous coordinates, this point corresponds to $(\infty, \infty)$, which is linearly equivalent to the vertex $V_{3}=(K, K)$. Similarly, the two points $[1:-\omega: 0],\left[1:-\omega^{2}: 0\right]$ also correspond to $V_{3}$. Furthermore, the triple of points $\left\{[1: 0:-1],[1: 0:-\omega],\left[1: 0:-\omega^{2}\right]\right\}$ correspond to $V_{2}=(0,-K)$, and the triple $\left\{[0: 1:-1],[0: 1:-\omega],\left[0: 1:-\omega^{2}\right]\right\}$ to $V_{1}=(-K, 0)$. In other words, three inflection points of the Hesse cubic curve degenerate to each vertex of $C_{K}$ in the ultradiscrete limit. This explains the reason why the multiplicity of each vertex of $C_{K}$ is three and $C_{K}$ is not smooth while the Hesse cubic curve is non-singular.

$\dagger$ It should be remarked that the term 'tropical' has been used differently in the communities of geometry and integrable systems [18]. In the former community it has been used to mean piecewise linear objects, while in the latter subtraction-free rational maps. In the latter community the terms 'crystal' or 'ultradiscrete' have been used for piecewise linear objects. Therefore, it sometimes happens that the term 'tropicalization' can be used with opposite meanings. 


\subsection{Ultradiscretization of general solution}

In this section, we consider the ultradiscrete limit of the solution. The following is the main result of this paper.

THEOREM 4.2. The general solution (3.8) of the rational map (3.5) reduces to the general solution (2.32) of the piecewise linear map (2.8) by taking the limit $\epsilon \rightarrow+0$ under the parametrization

$$
\begin{gathered}
X_{n}=e^{x_{n} / \epsilon}, \quad Y_{n}=e^{y_{n} / \epsilon}, \quad \frac{\tau}{\tau+\frac{1}{3}}=-\frac{9 K}{2 \pi i \epsilon}, \\
z_{0}=\frac{u_{0}}{9 K}\left(1-\frac{2 \pi i \epsilon}{9 K}\right), \quad u_{0} \in \mathbb{R}, \quad K>0 .
\end{gathered}
$$

The ultradiscrete limit of the theta function can be realized by taking $\operatorname{Im} \tau \rightarrow 0$. However, the limit of the real part of $\tau$ should be carefully chosen in order to obtain a consistent result [32]. For choosing the limit of the real part of $\tau$, the following observation on the correspondence between the zeros of the theta functions and non-smooth points of $S(u ; \alpha, \beta, \theta)$ is crucial.

ObSERVATION. The ultradiscrete theta function $\Theta(u ; \theta)$ defined by equation (2.29) is a piecewise quadratic function with period one, and has zeros at $u=n \in \mathbb{Z} ; \Theta(u ; \theta)$ can be obtained from $\vartheta_{0}(z ; \tau)=\vartheta_{(0,1 / 2)}(z ; \tau)$ by taking the limit $\tau \rightarrow 0$ [32]. Since the zeros of $\vartheta_{0}(z ; \tau)$ are located at $z=\left(m+\frac{1}{2}\right) \tau+n(m, n \in \mathbb{Z})$, the real zeros of $\vartheta_{0}(z ; \tau)$ survive under the limit, giving the zeros of $\Theta(u ; \theta)$ at $u=n$. From the definition of $S$ given in equation (2.30) and Figure 6, it is easy to see that the valleys at $u=n \alpha$ and the peaks at $u=\beta+n \alpha(n \in \mathbb{Z})$ of $S$ correspond to the zeros of $\Theta(u / \alpha ; \theta)$ and $\Theta((u-\beta) / \alpha ; \theta)$, respectively, as illustrated in Figure 10. In other words, the valleys and peaks of the ultradiscrete elliptic function $S$ arise from the zeros and poles of the corresponding elliptic function, respectively. Now, noticing that the zeros of $\vartheta_{(a, b)}(z, \tau)$ are located at $z=(-a+$ $\left.m+\frac{1}{2}\right) \tau+\left(-b+n+\frac{1}{2}\right)(m, n \in \mathbb{Z})$, the zeros of $\theta_{k}(z, \tau)(k=0,1,2)$ are given by

$$
\begin{array}{ll}
\theta_{0}(z, \tau): & z=\left(m+\frac{2}{3}\right) \tau+\frac{1}{3}(n-1), \\
\theta_{1}(z, \tau): & z=\left(m+\frac{1}{3}\right) \tau+\frac{1}{3}(n-1), \\
\theta_{2}(z, \tau): & z=m \tau+\frac{1}{3}(n-1),
\end{array}
$$

respectively. It is obvious that the zeros and poles of $x_{n}=\theta_{0}(z, \tau) / \theta_{2}(z, \tau)$ and $y_{n}=$ $\theta_{1}(z, \tau) / \theta_{2}(z, \tau)$ cancel each other, respectively, in the limit $\tau \rightarrow 0$, which yields a trivial result. Let us choose $\tau \rightarrow-\frac{1}{3}$. Then the zeros of $\theta_{i}(z, \tau)(i=0,1,2)$ become

$$
\begin{aligned}
& \theta_{0}(z, \tau): \quad z=\frac{\mathbb{Z}}{3}-\frac{2}{9}=\frac{\mathbb{Z}}{3}+\frac{1}{9}, \\
& \theta_{1}(z, \tau): \quad z=\frac{\mathbb{Z}}{3}-\frac{1}{9}=\frac{\mathbb{Z}}{3}+\frac{2}{9}, \\
& \theta_{2}(z, \tau): \quad z=\frac{\mathbb{Z}}{3},
\end{aligned}
$$

respectively, which give the zigzag patterns in the limit as illustrated in Figure 11. These patterns would coincide with the those in Figure 5 after an appropriate scaling. 


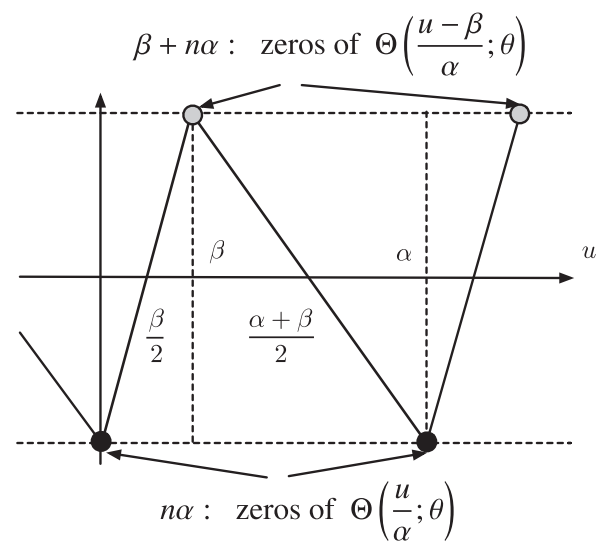

FIGURE 10. Zigzag pattern of $S(u ; \alpha, \beta, \theta)=\Theta(u / \alpha ; \theta)-\Theta((u-\beta) / \alpha ; \theta)$ and the zeros of $\Theta(u / \alpha ; \theta), \Theta((u-\beta) / \alpha ; \theta)$.
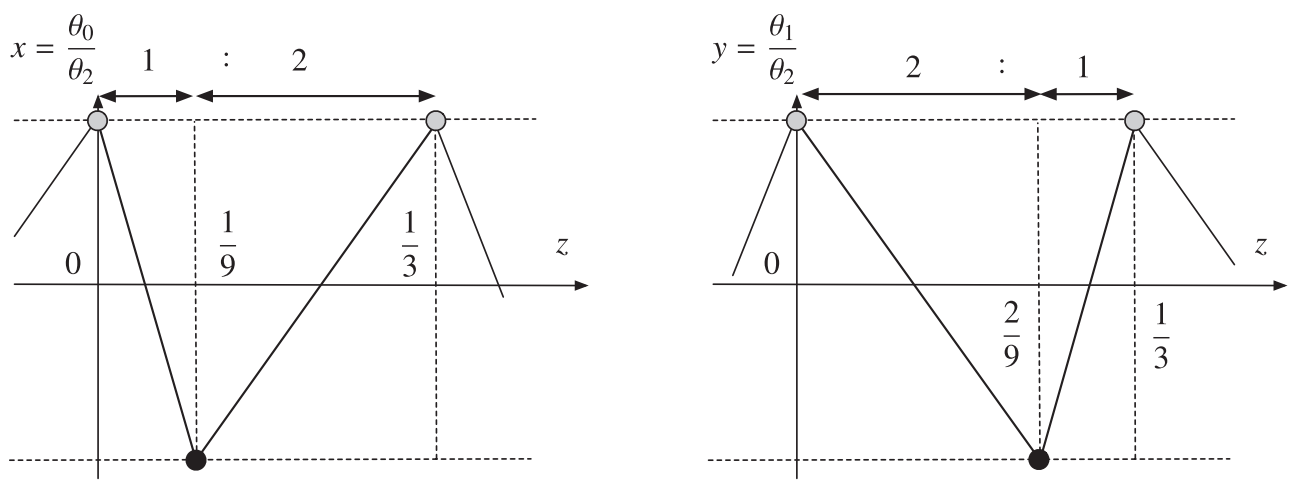

FIGURE 11. Zigzag patterns obtained by the limit $\tau \rightarrow-\frac{1}{3}$.

Before proceeding to the proof of Theorem 4.2, we prepare the modular transformation of the theta function, which is useful in taking the limit of $\tau$.

Proposition 4.3. [9, 28] The following holds:

$$
\vartheta_{\sigma \cdot m}(\sigma \cdot z, \sigma \cdot \tau)=e^{\pi i(\sigma \cdot z) c z}(c \tau+d)^{1 / 2} \kappa(\sigma) e^{2 \pi i \phi_{m}(\sigma)} \vartheta_{m}(z, \tau),
$$

where

$$
\left\{\begin{array}{l}
m=\left(m_{1}, m_{2}\right), \quad \sigma=\left(\begin{array}{ll}
a & b \\
c & d
\end{array}\right) \in \mathrm{SL}_{2}(\mathbb{Z}), \\
\sigma \cdot m=m \sigma^{-1}+\frac{1}{2}(c d, a b), \quad \sigma \cdot \tau=\frac{a \tau+b}{c \tau+d}, \quad \sigma \cdot z=\frac{z}{c \tau+d}, \\
\phi_{m}(\sigma)=-\frac{1}{2}\left[b d m_{1}^{2}+a c m_{2}^{2}-2 b c m_{1} m_{2}-a b\left(d m_{1}-c m_{2}\right)\right] \\
\kappa(\sigma): \text { an eighth root of } 1 \text { depending only on } \sigma .
\end{array}\right.
$$


Remark 4.4. The explicit expression of $\kappa(\sigma)$ is given by [8]

$$
\kappa(\sigma)= \begin{cases}e^{\pi i\left(a b c d / 2+a c d^{2} / 4-c / 4\right)}\left(\frac{a}{|c|}\right), & c \text { odd } \\ \left(\frac{c}{|d|}\right)(-1)^{(\operatorname{sgn}(c)-1)(\operatorname{sgn}(d)-1) / 4} e^{\pi i / 4(d-1)}, & c \text { even }\end{cases}
$$

where $\left(\frac{a}{p}\right)$ is the Legendre (Jacobi) symbol for the quadratic residue. In particular, $\left(\frac{a}{1}\right)=1$.

Proof of Theorem 4.2. The first key part of the proof is to apply the modular transformation on $\theta_{k}(z, \tau)=\vartheta_{(k / 3-1 / 6,3 / 2)}(3 z, 3 \tau)(k=0,1,2)$ specified by

$$
\sigma=\left(\begin{array}{ll}
1 & 0 \\
1 & 1
\end{array}\right)
$$

From

$$
\sigma \cdot m=\left(\frac{k}{3}-\frac{7}{6}, \frac{3}{2}\right), \quad \phi_{m}(\sigma)=-\frac{9}{8}, \quad \kappa(\sigma)=1,
$$

we have

$$
\begin{aligned}
\theta_{k}(z, \tau) & =\vartheta_{(k / 3-1 / 6,3 / 2)}(3 z, 3 \tau) \\
& =e^{-3 \pi i z^{2} /(\tau+1 / 3)}(3 \tau+1)^{-1 / 2} e^{\pi i / 4} \vartheta_{(k / 3-7 / 6,3 / 2)}\left(\frac{z}{\tau+\frac{1}{3}}, \frac{\tau}{\tau+\frac{1}{3}}\right) .
\end{aligned}
$$

We put

$$
\frac{\tau}{\tau+\frac{1}{3}}=-\frac{\theta}{i \pi \epsilon}, \quad \theta>0
$$

and take the limit of $\epsilon \rightarrow+0$, which corresponds to $\tau \rightarrow-\frac{1}{3}$. Noting that

$$
\frac{z}{\tau+\frac{1}{3}}=3\left(\frac{\theta}{i \pi \epsilon}+1\right) z
$$

we have

$$
\begin{aligned}
\vartheta_{(k / 3-7 / 6,3 / 2)}\left(\frac{z}{\tau+\frac{1}{3}}, \frac{\tau}{\tau+\frac{1}{3}}\right)= & \sum_{n \in \mathbb{Z}} \exp \left[-\frac{\theta}{\epsilon}\left(n+\frac{k}{3}-\frac{7}{6}\right)^{2}+\frac{6 \theta}{\epsilon}\left(n+\frac{k}{3}-\frac{7}{6}\right) z\right] \\
& \times \exp \left[6 \pi i\left(n+\frac{k}{3}-\frac{7}{6}\right)\left(z+\frac{1}{2}\right)\right] .
\end{aligned}
$$

The second key part is to use the freedom of the imaginary part of $z \in \mathbb{C}$. Putting

$$
z=\frac{u}{9 K}+i v, \quad K, u, v \in \mathbb{R}
$$


equation (4.15) is rewritten as

$$
\begin{aligned}
\vartheta_{(k / 3-7 / 6,3 / 2)}\left(\frac{z}{\tau+\frac{1}{3}}, \frac{\tau}{\tau+\frac{1}{3}}\right) \\
=\sum_{n \in \mathbb{Z}} \exp \left[-\frac{\theta}{\epsilon}\left(n+\frac{k}{3}-\frac{7}{6}\right)^{2}+\frac{6 \theta}{\epsilon}\left(n+\frac{k}{3}-\frac{7}{6}\right)\left(\frac{u}{9 K}+i v\right)\right] \\
\quad \times \exp \left[6 \pi i\left(n+\frac{k}{3}-\frac{7}{6}\right)\left(\frac{u}{9 K}+i v+\frac{1}{2}\right)\right] \\
=\exp \left(\frac{\theta}{9 K^{2} \epsilon} u^{2}\right) \sum_{n \in \mathbb{Z}} \exp \left[-\frac{\theta}{\epsilon}\left(\frac{u}{3 K}-n-\frac{k}{3}+\frac{7}{6}\right)^{2}-6 \pi\left(n+\frac{k}{3}-\frac{7}{6}\right) v\right] \\
\quad \times \exp \left[6 \pi i\left(n+\frac{k}{3}-\frac{7}{6}\right)\left(\frac{\theta}{\pi \epsilon} v+\frac{u}{9 K}+\frac{1}{2}\right)\right] .
\end{aligned}
$$

If $u$ and $v$ satisfy

$$
\frac{\theta}{\pi \epsilon} v+\frac{u}{9 K}=0
$$

then equation (4.17) is simplified as

$$
\begin{aligned}
\left.\vartheta_{(k / 3}-7 / 6,3 / 2\right) & \left(\frac{z}{\tau+\frac{1}{3}}, \frac{\tau}{\tau+\frac{1}{3}}\right) \\
= & \exp \left(\frac{\theta}{9 K^{2} \epsilon} u^{2}\right) \exp \left[\pi i\left(k-\frac{7}{2}\right)\right] \\
& \times \sum_{n \in \mathbb{Z}} \exp \left[-\frac{\theta}{\epsilon}\left(\frac{u}{3 K}-n-\frac{k}{3}+\frac{7}{6}\right)^{2}-6 \pi\left(n+\frac{k}{3}-\frac{7}{6}\right) v\right] e^{3 \pi i n} \\
= & \exp \left(\frac{\theta}{9 K^{2} \epsilon} u^{2}\right) \exp \left[\pi i\left(k-\frac{7}{2}\right)\right] \\
& \times \sum_{n \in \mathbb{Z}} \exp \left[-\frac{\theta}{\epsilon}\left(\frac{u-K(k+1)}{3 K}-(n-2)-\frac{1}{2}\right)^{2}+\left(n+\frac{k}{3}-\frac{7}{6}\right) \frac{2 \pi^{2} \epsilon}{3 K \theta} u\right] e^{3 \pi i n} .
\end{aligned}
$$

Therefore, the asymptotic behaviors of $x_{n}$ and $y_{n}$ as $\epsilon \rightarrow+0$ are given by

$$
\begin{aligned}
& x_{n}=\frac{\theta_{0}(z, \tau)}{\theta_{2}(z, \tau)} \sim \frac{\sum_{n \in \mathbb{Z}} \exp \left[-\frac{\theta}{\epsilon}\left(\frac{u-K}{3 K}-(n-2)-\frac{1}{2}\right)^{2}\right] e^{3 n \pi i}}{e^{2 \pi i} \sum_{n \in \mathbb{Z}} \exp \left[-\frac{\theta}{\epsilon}\left(\frac{u-3 K}{3 K}-\frac{1}{2}-(n-2)\right)^{2}\right] e^{3 n \pi i}} \\
& \sim e^{\left(n_{0}+n_{2}\right) \pi i} \frac{\exp \left\{-\frac{\theta}{\epsilon}\left[\left(\left(\frac{u-K}{3 K}\right)\right)-\frac{1}{2}\right]^{2}\right\}}{\exp \left\{-\frac{\theta}{\epsilon}\left[\left(\left(\frac{u-3 K}{3 K}\right)\right)-\frac{1}{2}\right]^{2}\right\}}, \\
& y_{n}=\frac{\theta_{1}(z, \tau)}{\theta_{2}(z, \tau)} \sim \frac{e^{\pi i} \sum_{n \in \mathbb{Z}} \exp \left[-\frac{\theta}{\epsilon}\left(\frac{u-2 K}{3 K}-(n-2)-\frac{1}{2}\right)^{2}\right] e^{3 n \pi i}}{e^{2 \pi i} \sum_{n \in \mathbb{Z}} \exp \left[-\frac{\theta}{\epsilon}\left(\frac{u-3 K}{3 K}-(n-2)-\frac{1}{2}\right)^{2}\right] e^{3 n \pi i}} \\
& \sim e^{\left(n_{1}+n_{2}+1\right) \pi i} \frac{\exp \left\{-\frac{\theta}{\epsilon}\left[\left(\left(\frac{u-2 K}{3 K}\right)\right)-\frac{1}{2}\right]^{2}\right\}}{\exp \left\{-\frac{\theta}{\epsilon}\left[\left(\left(\frac{u-3 K}{3 K}\right)\right)-\frac{1}{2}\right]^{2}\right\}},
\end{aligned}
$$


respectively, where $((u))(u \in \mathbb{R})$ is defined in equation (2.29), and

$$
n_{0}=\text { Floor }\left[\frac{u-K}{3 K}\right]+2, \quad n_{1}=\text { Floor }\left[\frac{u-2 K}{3 K}\right]+2, \quad n_{2}=\text { Floor }\left[\frac{u-3 K}{3 K}\right]+2 .
$$

Here, we note that $z$ and $u$ are understood as $2^{n} z_{0}$ and $2^{n} u_{0}$, respectively.

In order to obtain the final result, the remaining task is to relate the parameters $\theta$ with $K$ in the limit $\epsilon \rightarrow+0$. This can be done by considering the limit of the conserved quantity $\mu(\tau)$ given by equation (3.9) or (3.40). Noting equation (3.40), we differentiate $\theta_{0}(z, \tau)$ and $\theta_{2}(z, \tau)$ with respect to $z$ after applying the modular transformation. Then $\theta_{0}^{\prime}(0, \tau)$ and $\theta_{1}^{\prime}(0, \tau)$ can be calculated by using equations (4.13) and (4.15) as

$$
\begin{aligned}
\theta_{0}^{\prime}(0, & \tau)=\left.\frac{\partial}{\partial z} \vartheta_{(-1 / 6,3 / 2)}(3 z, 3 \tau)\right|_{z=0} \\
= & \frac{\partial}{\partial z} \exp \left[-\pi i\left(1+\frac{\theta}{i \pi \epsilon}\right) z^{2}\right]\left(1+\frac{\theta}{i \pi \epsilon}\right)^{1 / 2} e^{\pi i / 4} \\
& \times\left.\sum_{n \in \mathbb{Z}} \exp \left[-\frac{\theta}{\epsilon}\left(n-\frac{7}{6}\right)^{2}+\frac{6 \theta}{\epsilon}\left(n-\frac{7}{6}\right) z\right] \exp \left[6 \pi i\left(n-\frac{7}{6}\right)\left(z+\frac{1}{2}\right)\right]\right|_{z=0} \\
= & \left(1+\frac{\theta}{i \pi \epsilon}\right)^{1 / 2} e^{3 \pi i / 4} \sum_{n \in \mathbb{Z}}\left[6\left(\pi i+\frac{\theta}{\epsilon}\right)\left(n-\frac{7}{6}\right)\right] \exp \left[-\frac{\theta}{\epsilon}\left(n-\frac{7}{6}\right)^{2}+3 n \pi i\right], \\
\theta_{2}^{\prime}(0, & \tau)=\left.\frac{\partial}{\partial z} \vartheta_{(1 / 2,3 / 2)}(3 z, 3 \tau)\right|_{z=0} \\
= & \frac{\partial}{\partial z} \exp \left[-\pi i\left(1+\frac{\theta}{i \pi \epsilon}\right) z^{2}\right]\left(1+\frac{\theta}{i \pi \epsilon}\right)^{1 / 2} e^{\pi i / 4} \\
& \times\left.\sum_{n \in \mathbb{Z}} \exp \left[-\frac{\theta}{\epsilon}\left(n-\frac{1}{2}\right)^{2}+\frac{6 \theta}{\epsilon}\left(n-\frac{1}{2}\right) z\right] \exp \left[6 \pi i\left(n-\frac{1}{2}\right)\left(z+\frac{1}{2}\right)\right]\right|_{z=0} \\
= & \left(1+\frac{\theta}{i \pi \epsilon}\right)^{1 / 2} e^{3 \pi i / 4} \sum_{n \in \mathbb{Z}}\left[6\left(\pi i+\frac{\theta}{\epsilon}\right)\left(n-\frac{1}{2}\right)\right] \exp \left[-\frac{\theta}{\epsilon}\left(n-\frac{1}{2}\right)^{2}+3 n \pi i\right],
\end{aligned}
$$

respectively, which imply

$$
\begin{aligned}
3 \mu & =-6 \frac{\theta_{0}^{\prime}(0, \tau)}{\theta_{2}^{\prime}(0, \tau)}=-6 \frac{e^{3 \pi i / 4} \sum_{n \in \mathbb{Z}}\left(n-\frac{7}{6}\right) \exp \left[-\frac{\theta}{\epsilon}\left(n-\frac{7}{6}\right)^{2}+3 n \pi i\right]}{e^{3 \pi i / 4} \sum_{n \in \mathbb{Z}}\left(n-\frac{1}{2}\right) \exp \left[-\frac{\theta}{\epsilon}\left(n-\frac{1}{2}\right)^{2}+3 n \pi i\right]} \\
& \sim-6 \frac{\left(-\frac{1}{6}\right) \exp \left(-\frac{\theta}{36 \epsilon}+3 \pi i\right)}{-\frac{1}{2} \exp \left(-\frac{\theta}{4 \epsilon}\right)+\frac{1}{2} \exp \left(-\frac{\theta}{4 \epsilon}+3 \pi i\right)}=e^{2 \theta / 9 \epsilon},
\end{aligned}
$$

as $\epsilon \rightarrow+0$. Accordingly, from equation (4.6) we may put consistently

$$
\theta=\frac{9}{2} K
$$

Let us set $x_{n}=e^{X_{n} / \epsilon}$ and $y_{n}=e^{Y_{n} / \epsilon}$ in equation (4.19). Then the complex factors in equation (4.19) disappear in the limit of $\epsilon \rightarrow+0$ and we finally obtain by using 
TABLE 1. Quadrant of $\left(x_{n}, y_{n}\right)$ for $\epsilon \sim+0$, where $N=\operatorname{Floor}\left(\left(\frac{u}{3 K}\right)\right)+2$.

\begin{tabular}{ccccccc}
\hline$\left(\left(\frac{u}{3 K}\right)\right)$ & $n_{0}$ & $n_{1}$ & $n_{2}$ & $n_{0}+n_{2}$ & $n_{1}+n_{2}+1$ & $\left(x_{n}, y_{n}\right)$ \\
\hline$\left[\frac{2}{3}, 1\right)$ & $N$ & $N$ & $N-1$ & $2 N-1$ & $2 N$ & $(-,+)$ \\
{$\left[\frac{1}{3}, \frac{2}{3}\right)$} & $N$ & $N-1$ & $N-1$ & $2 N-1$ & $2 N-1$ & $(-,-)$ \\
{$\left[0, \frac{1}{3}\right)$} & $N-1$ & $N-1$ & $N-1$ & $2 N-2$ & $2 N-1$ & $(+,-)$ \\
\hline
\end{tabular}

equation (4.21)

$$
\begin{aligned}
& X_{n}=-\frac{9 K}{2}\left[\left(\left(\frac{u-K}{3 K}\right)\right)-\frac{1}{2}\right]^{2}+\frac{9 K}{2}\left[\left(\left(\frac{u-3 K}{3 K}\right)\right)-\frac{1}{2}\right]^{2}, \\
& Y_{n}=-\frac{9 K}{2}\left[\left(\left(\frac{u-2 K}{3 K}\right)\right)-\frac{1}{2}\right]^{2}+\frac{9 K}{2}\left[\left(\left(\frac{u-3 K}{3 K}\right)\right)-\frac{1}{2}\right]^{2},
\end{aligned}
$$

which is equivalent to equation (2.32). This completes the proof.

We finally remark that the choice of parametrization (4.8) can also be justified by the following observation. The asymptotic formula (4.19) shows that $\left(x_{n}, y_{n}\right)$ is in $\mathbb{R}^{2}$ and that the quadrant of $\left(x_{n}, y_{n}\right)$ changes according to the value of $\left(\left(\frac{u}{3 K}\right)\right)$ as described in Table 1 . Note that $\left(x_{n}, y_{n}\right)$ never enters the first quadrant. It implies that the qualitative behavior of the real orbit of the map (2.8) discussed in Section 3 is preserved under the limiting process.

Acknowledgements. The authors would like to express their sincere thanks to Professor A. Nakayashiki and Professor Y. Yamada for fruitful discussions. This work was partially supported by the JSPS Grants-in-Aid for Scientific Research Nos. 19340039, 19740086 and 19840039.

\section{A. Proof of Lemma 3.3}

In this appendix, we give a proof of Lemma 3.3. Besides the cases $k=0,1,2$, we also use the cases $k=\frac{1}{2}, \frac{3}{2}, \frac{5}{2}$ as well. Note that $\theta_{k+3}(z, \tau)=\theta_{k}(z, \tau)$.

First, we remark that $\theta_{1 / 2}(z, \tau)=\vartheta_{(0,3 / 2)}(3 z, 3 \tau)$ and $\theta_{2}(z, \tau)=\vartheta_{(1 / 2,3 / 2)}(3 z, 3 \tau)$ are $\vartheta(3 z, 3 \tau)$ and $\vartheta_{1}(3 z, 3 \tau)$ of Jacobi's notation, respectively. Let us start from equation (A)-(4) in [14]:

$$
\begin{aligned}
& \theta_{1 / 2}(w) \theta_{1 / 2}(x) \theta_{1 / 2}(y) \theta_{1 / 2}(z)-\theta_{2}(w) \theta_{2}(x) \theta_{2}(y) \theta_{2}(z) \\
& \quad=\theta_{1 / 2}\left(w^{\prime}\right) \theta_{1 / 2}\left(x^{\prime}\right) \theta_{1 / 2}\left(y^{\prime}\right) \theta_{1 / 2}\left(z^{\prime}\right)-\theta_{2}\left(w^{\prime}\right) \theta_{2}\left(x^{\prime}\right) \theta_{2}\left(y^{\prime}\right) \theta_{2}\left(z^{\prime}\right),
\end{aligned}
$$

where

$$
\begin{array}{ll}
w^{\prime}=\frac{w+x+y+z}{2}, & x^{\prime}=\frac{w+x-y-z}{2}, \\
y^{\prime}=\frac{w-x+y-z}{2}, & z^{\prime}=\frac{w-x-y+z}{2} .
\end{array}
$$


Replacing $w \rightarrow w+\tau / 3$, we have $w^{\prime} \rightarrow w^{\prime}+\tau / 6, x^{\prime} \rightarrow x^{\prime}+\tau / 6, y^{\prime} \rightarrow y^{\prime}+\tau / 6$ and $z^{\prime} \rightarrow z^{\prime}+\tau / 6$. By using the formula

$$
\theta_{k}\left(z+\frac{\tau}{6}\right)=e^{-\pi i / 2} e^{-\pi i \tau / 12-\pi i z} \theta_{k+1 / 2}(z), \quad \theta_{k}\left(z+\frac{\tau}{3}\right)=e^{-\pi i} e^{-\pi i \tau / 3-2 \pi i z} \theta_{k+1}(z),
$$

which easily follows by definition, we obtain

$$
\begin{aligned}
- & \theta_{3 / 2}(w) \theta_{1 / 2}(x) \theta_{1 / 2}(y) \theta_{1 / 2}(z)+\theta_{0}(w) \theta_{2}(x) \theta_{2}(y) \theta_{2}(z) \\
& =\theta_{1}\left(w^{\prime}\right) \theta_{1}\left(x^{\prime}\right) \theta_{1}\left(y^{\prime}\right) \theta_{1}\left(z^{\prime}\right)-\theta_{5 / 2}\left(w^{\prime}\right) \theta_{5 / 2}\left(x^{\prime}\right) \theta_{5 / 2}\left(y^{\prime}\right) \theta_{5 / 2}\left(z^{\prime}\right) .
\end{aligned}
$$

Replacing further $w \rightarrow w+\tau / 3, x \rightarrow x+\tau / 3, y \rightarrow y+\tau / 3$ and $z \rightarrow z+\tau / 3$, we see that $w^{\prime} \rightarrow w^{\prime}+2 \tau / 3$ and $x^{\prime}, y^{\prime}, z^{\prime}$ are unchanged. Then we obtain

$$
\begin{aligned}
- & \theta_{5 / 2}(w) \theta_{3 / 2}(x) \theta_{3 / 2}(y) \theta_{3 / 2}(z)+\theta_{1}(w) \theta_{0}(x) \theta_{0}(y) \theta_{0}(z) \\
& =\theta_{0}\left(w^{\prime}\right) \theta_{1}\left(x^{\prime}\right) \theta_{1}\left(y^{\prime}\right) \theta_{1}\left(z^{\prime}\right)-\theta_{3 / 2}\left(w^{\prime}\right) \theta_{5 / 2}\left(x^{\prime}\right) \theta_{5 / 2}\left(y^{\prime}\right) \theta_{5 / 2}\left(z^{\prime}\right) .
\end{aligned}
$$

Application of the same transformation to equation (A.1) yields

$$
\begin{aligned}
& \theta_{3 / 2}(w) \theta_{3 / 2}(x) \theta_{3 / 2}(y) \theta_{3 / 2}(z)-\theta_{0}(w) \theta_{0}(x) \theta_{0}(y) \theta_{0}(z) \\
& =\theta_{5 / 2}\left(w^{\prime}\right) \theta_{1 / 2}\left(x^{\prime}\right) \theta_{1 / 2}\left(y^{\prime}\right) \theta_{1 / 2}\left(z^{\prime}\right)-\theta_{1}\left(w^{\prime}\right) \theta_{2}\left(x^{\prime}\right) \theta_{2}\left(y^{\prime}\right) \theta_{2}\left(z^{\prime}\right) .
\end{aligned}
$$

We put $w=-(x+y+z)$ in equations (A.4) and (A.5). Then $w^{\prime}=0, x^{\prime}=-(y+z)$, $y^{\prime}=-(z+x)$ and $z^{\prime}=-(x+y)$. By definition it follows that

$$
\theta_{0}(-z)=-\theta_{1}(z), \quad \theta_{1 / 2}(-z)=\theta_{1 / 2}(z), \quad \theta_{2}(-z)=-\theta_{2}(z), \quad \theta_{3 / 2}(-z)=\theta_{5 / 2}(z),
$$

and hence

$$
\theta_{1}(0)=-\theta_{0}(0), \quad \theta_{5 / 2}(0)=\theta_{3 / 2}(0), \quad \theta_{2}(0)=0 .
$$

Therefore equations (A.4) and (A.5) yield

$$
\begin{aligned}
& -\theta_{5 / 2}(x+y+z) \theta_{1 / 2}(x) \theta_{1 / 2}(y) \theta_{1 / 2}(z)-\theta_{1}(x+y+z) \theta_{2}(x) \theta_{2}(y) \theta_{2}(z) \\
& \quad=\theta_{1}(0) \theta_{0}(y+z) \theta_{0}(z+x) \theta_{0}(x+y)-\theta_{3 / 2}(0) \theta_{3 / 2}(y+z) \theta_{3 / 2}(z+x) \theta_{3 / 2}(x+y), \\
& \theta_{3 / 2}(x+y+z) \theta_{3 / 2}(x) \theta_{3 / 2}(y) \theta_{3 / 2}(z)+\theta_{0}(x+y+z) \theta_{0}(x) \theta_{0}(y) \theta_{0}(z) \\
& \quad=-\theta_{0}(0) \theta_{0}(y+z) \theta_{0}(z+x) \theta_{0}(x+y)-\theta_{3 / 2}(0) \theta_{3 / 2}(y+z) \theta_{3 / 2}(z+x) \theta_{3 / 2}(x+y),
\end{aligned}
$$

respectively. Similarly, putting $w=x+y+z$, we have that $w^{\prime}=x+y+z$ and $x^{\prime}, y^{\prime}, z^{\prime}$ are unchanged. Then equation (A.6) yields

$$
\begin{aligned}
& \theta_{3 / 2}(x+y+z) \theta_{3 / 2}(x) \theta_{3 / 2}(y) \theta_{3 / 2}(z)-\theta_{0}(x+y+z) \theta_{0}(x) \theta_{0}(y) \theta_{0}(z) \\
& \quad=\theta_{5 / 2}(x+y+z) \theta_{1 / 2}(x) \theta_{1 / 2}(y) \theta_{1 / 2}(z)-\theta_{1}(x+y+z) \theta_{2}(x) \theta_{2}(y) \theta_{2}(z) .
\end{aligned}
$$

Then from adding - (A.9) + (A.10) + (A.11) and dividing it by 2 , we have

$$
\begin{aligned}
& \theta_{0}(0) \theta_{0}(y+z) \theta_{0}(z+x) \theta_{0}(x+y) \\
& \quad=\theta_{0}(x+y+z) \theta_{0}(x) \theta_{0}(y) \theta_{0}(z)-\theta_{1}(x+y+z) \theta_{2}(x) \theta_{2}(y) \theta_{2}(z),
\end{aligned}
$$


which yields equation (3.12) by putting $z=-y$ in equation (A.12). Other addition formulas are derived from equation (3.12). Applying $x \rightarrow x+\tau / 3, y \rightarrow y+\tau / 3$ on equation (3.12), we have

$$
\theta_{0}(0)^{2} \theta_{1}(x+y) \theta_{0}(x-y)=\theta_{0}(x) \theta_{1}(x) \theta_{1}(y)^{2}-\theta_{2}(x)^{2} \theta_{0}(y) \theta_{2}(y) .
$$

Repeating the same procedure on equation (A.13), we obtain

$$
\theta_{0}(0)^{2} \theta_{2}(x+y) \theta_{0}(x-y)=\theta_{0}(x) \theta_{2}(x) \theta_{0}(y)^{2}-\theta_{1}(x)^{2} \theta_{1}(y) \theta_{2}(y) .
$$

Exchanging $x \leftrightarrow y$ in equation (3.12), we have

$$
\theta_{0}(0)^{2} \theta_{0}(x+y) \theta_{1}(x-y)=\theta_{0}(x) \theta_{1}(x) \theta_{0}(y)^{2}-\theta_{2}(x)^{2} \theta_{1}(y) \theta_{2}(y) .
$$

Repeating $x \rightarrow x+\tau / 3, y \rightarrow y+\tau / 3$ on equation (A.15) twice yields

$$
\begin{aligned}
& \theta_{0}(0)^{2} \theta_{1}(x+y) \theta_{1}(x-y)=\theta_{0}(x) \theta_{2}(x) \theta_{2}(y)^{2}-\theta_{1}(x)^{2} \theta_{0}(y) \theta_{1}(y), \\
& \theta_{0}(0)^{2} \theta_{2}(x+y) \theta_{1}(x-y)=\theta_{1}(x) \theta_{2}(x) \theta_{1}(y)^{2}-\theta_{0}(x)^{2} \theta_{0}(y) \theta_{2}(y),
\end{aligned}
$$

respectively. Applying $x \rightarrow x+\tau / 3$ to equation (A.17), we obtain

$$
\theta_{0}(0)^{2} \theta_{0}(x+y) \theta_{2}(x-y)=\theta_{0}(x) \theta_{2}(x) \theta_{1}(y)^{2}-\theta_{1}(x)^{2} \theta_{0}(y) \theta_{2}(y) .
$$

Again, repeating $x \rightarrow x+\tau / 3, y \rightarrow y+\tau / 3$ on equation (A.15) twice, we have

$$
\begin{aligned}
& \theta_{0}(0)^{2} \theta_{1}(x+y) \theta_{2}(x-y)=\theta_{1}(x) \theta_{2}(x) \theta_{0}(y)^{2}-\theta_{0}(x)^{2} \theta_{1}(y) \theta_{2}(y), \\
& \theta_{0}(0)^{2} \theta_{2}(x+y) \theta_{2}(x-y)=\theta_{0}(x) \theta_{1}(x) \theta_{2}(y)^{2}-\theta_{2}(x)^{2} \theta_{0}(y) \theta_{1}(y),
\end{aligned}
$$

respectively. This completes the proof of Lemma 3.3.

\section{REFERENCES}

[1] M. Artebani and I. Dolgachev. The Hesse pencil of plane cubic curves. Preprint, arXiv:math/0611590v3 [math.AG], 2006.

[2] C. Birkenhake and H. Lange. Cubic theta relation. J. Reine Angew. Math. 407 (1990), 167-177.

[3] A. Gathmann. Tropical algebraic geometry. Preprint, arXiv:math/0601322v1 [math.AG], 2006.

[4] B. Grammaticos, A. Ramani and C. M. Viallet. Solvable chaos. Phys. Lett. A336 (2005), 152-158.

[5] G. Hatayama, K. Hikami, R. Inoue, A. Kuniba, T. Takagi and T. Tokihiro. The $A_{M}^{(1)}$ automata related to crystals of symmetric tensors. J. Math. Phys. 42 (2001), 274-308.

[6] K. Hikami, R. Inoue and Y. Komori. Crystallization of the Bogoyavlensky lattice. J. Phys. Soc. Jpn. 68 (1999), 2234-2240.

[7] R. Hirota and D. Takahashi. Ultradiscretization of the Tzitzeica equation. Glasgow Math. J. 47 (2005), 7785 .

[8] T. Ibukiyama. Modular forms of rational weights. Introduction to modular forms of half integral weights. Proceedings of the 8th Summer School on Number Theory, 2001, pp. 1-22 (in Japanese).

[9] J. Igusa. Theta Functions. Springer, Berlin, 1972.

[10] R. Inoue and T. Takenawa. Tropical spectral curves and integrable cellular automata. Int. Math. Res. Notices 2008 (2008), article ID rnn019.

[11] S. Isojima, M. Murata, A. Nobe and J. Satsuma. An ultradiscretization of the sine-Gordon equation. Phys. Lett. A331 (2004), 378-386.

[12] I. Itenberg, G. Mikhalkin and E. Shustin. Tropical Algebraic Feometry. Birkhäuser, Basel, 2007.

[13] S. Iwao and T. Tokihiro. Ultradiscretization of the theta function solution of pd Toda. J. Phys. A: Math. Theor. 40 (2007), 12987-13021. 
[14] C. G. J. Jacobi. Theorie der Elliptischen Funktionen aus den Eigenscheften der Thetareihen abgeleitet. Gesammelte Werke, Vol. I. G. Reimer, Berlin, 1881, p. 497.

[15] M. Joye and J.-J. Quisquater. Hessian elliptic curves and side-channel attacks. Cryptographic Hardware and Embedded Systems - CHES 2001 (Lecture Notes in Computer Science, 2162). Eds. Ç. K. Koç, D. Naccache and C. Paar. Springer, Berlin, 2001, pp. 402-410.

[16] K. Kajiwara, A. Nobe and T. Tsuda. Ultradiscretization of solvable one-dimensional chaotic maps. J. Phys. A: Math. Theor. 41 (2008), 395202 (13pp).

[17] T. Kimijima and T. Tokihiro. Initial-value problem of the discrete periodic Toda equation and its ultradiscretization. Inverse Problems 18 (2002), 1705-1732.

[18] A. N. Kirillov. Introduction to tropical combinatorics. Physics and Combinatorics 2000. Eds. A. N. Kirillov and N. Liskova. World Scientific, River Edge, NJ, 2001, pp. 82-150.

[19] A. Kuniba, M. Okado, R. Sakamoto, T. Takagi and Y. Yamada. Crystal interpretation of Kerov-KirillovReshetikhin bijection. Nucl. Phys. B740 (2006), 299-327.

[20] A. Kuniba and R. Sakamoto. Combinatorial Bethe ansatz and ultradiscrete Riemann theta function with rational characteristics. Lett. Math. Phys. 80 (2007), 199-209.

[21] A. Kuniba, R. Sakamoto and Y. Yamada. Tau functions in combinatorial Bethe ansatz. Nucl. Phys. B786 (2007), 207-266.

[22] A. Kuniba, T. Takagi and A. Takenouchi. Bethe ansatz and inverse scattering transform in a periodic box-ball system. Nucl. Phys. B747 (2006), 354-397.

[23] J. Matsukidaira, J. Satsuma, D. Takahashi, T. Tokihiro and M. Torii. Toda-type cellular automaton and its $N$-soliton solution. Phys. Lett. A225 (1997), 287-295.

[24] G. Mikhalkin. Enumerative tropical algebraic geometry in $\mathbb{R}^{2}$. J. Amer. Math. Soc. 18 (2005), 313-377.

[25] G. Mikhalkin. Tropical geometry and its applications. Preprint, arXiv:math/0601041v2 [math.AG], 2006.

[26] G. Mikhalkin and I. Zharkov. Tropical curves, their Jacobians and theta functions. Preprint, arXiv:math/0612267v2 [math.AG], 2006.

[27] J. Matsukidaira and K. Nishinari. Euler-Lagrange correspondence of cellular automaton for traffic-flow models. Phys. Rev. Lett. 90 (2003), 088701.

[28] D. Mumford. Tata Lectures on Theta I. Birkhäuser, Boston, 1983.

[29] H. Nagai. A new expression of a soliton solution to the ultradiscrete Toda equation. J. Phys. A: Math. Theor. 41 (2008), 235204 (12pp).

[30] K. Nishinari, J. Matsukidaira and D. Takahashi. Two-dimensional Burgers cellular automaton. J. Phys. Soc. Japan 70 (2001), 2267-2272.

[31] K. Nishinari and D. Takahashi. Analytical properties of ultradiscrete Burgers equation and rule-184 cellular automaton. J. Phys. A: Math. Gen. 31 (1998), 5439-5450.

[32] A. Nobe. Ultradiscretization of elliptic functions and its applications to integrable systems. J. Phys. A: Math. Gen. 39 (2006), L335-L342.

[33] A. Nobe. Ultradiscrete QRT maps and tropical elliptic curves. J. Phys. A: Math. Theor. 41 (2008), 125205 (12pp).

[34] J. Richter-Gebert, B. Sturmfels and T. Theobald. First steps in tropical geometry. Preprint, arXiv:math/0306366v2 [math.AG], 2003.

[35] N. P. Smart. The Hessian form of an elliptic curve. Cryptographic Hardware and Embedded Systems - CHES 2001 (Lecture Notes in Computer Science, 2162). Eds. Ç. K. Koç, D. Naccache and C. Paar. Springer, Berlin, 2001, pp. 118-125.

[36] D. Takahashi and R. Hirota. Ultradiscrete soliton solution of permanent type. J. Phys. Soc. Japan 76 (2007), 104007.

[37] D. Takahashi and J. Matsukdaira. Box and ball system with a carrier and ultradiscrete modified KdV equation. J. Phys. A: Math. Gen. 30 (1997), L733-L739.

[38] D. Takahashi and J. Matsukidaira. On a discrete optimal velocity model and its continuous and ultradiscrete relatives. Preprint, arXiv:0809.1265v1 [nlin.AO], 2008.

[39] D. Takahashi and J. Satsuma. A soliton cellular automaton. J. Phys. Soc. Japan 59 (1990), 3514-3519.

[40] D. Takahashi, A. Shida and M. Usami. On the pattern formation mechanism of $(2+1) \mathrm{D}$ max-plus models. J. Phys. A: Math. Gen. 34 (2001), 10715-10726.

[41] T. Tokihiro, D. Takahashi, J. Matsukidaira and J. Satsuma. From soliton equations to integrable cellular automata through a limiting procedure. Phys. Rev. Lett. 76 (1996), 3247-3250.

[42] D. Takahashi, T. Tokihiro, B. Grammaticos, Y. Ohta and A. Ramani. Constructing solutions to the ultradiscrete Painlevé equations. J. Phys. A: Math. Gen. 30 (1997), 7953-7966. 
[43] T. Tokihiro and J. Mada. Fundamental cycle of a periodic box-ball system: a number theoretical aspect. Glasgow Math. J. 47 (2005), 199-204.

[44] T. Tokihiro, D. Takahashi and J. Matsukidaira. Box and ball system as a realization of ultradiscrete nonautonomous KP equation. J. Phys. A: Math. Gen. 33 (2000), 607-619.

[45] T. Tsuda. Tropical Weyl group action via point configurations and $\tau$-functions of the $q$-Painlevé equations. Lett. Math. Phys. 77 (2006), 21-30.

[46] T. Tsuda and T. Takenawa. Tropical representation of Weyl groups associated with certain rational varieties. Adv. in Math. 221 (2009), 936-954.

[47] S. Tsujimoto and R. Hirota. Ultradiscrete KdV equation. J. Phys. Soc. Japan 67 (1998), 1809-1810.

[48] M. D. Vigeland. The group law on a tropical elliptic curve. Preprint, arXiv:math/0411485v1 [math.AG], 2004.

[49] Y. Yamada. An illustrated guide to the amoebae of type E - collection and observation. Talk delivered at Colloquium of Institute of Physics, Graduate School of Liberal Arts and Sciences, University of Tokyo, 2007.

[50] D. Yoshihara, F. Yura and T. Tokihiro. Fundamental cycle of a periodic box-ball system. J. Phys. A: Math. Gen. 36 (2003), 99-121.

\title{
Kenji Kajiwara, Masanobu Kaneko and Teruhisa Tsuda Faculty of Mathematics \\ Kyushu University \\ 6-10-1 Hakozaki \\ Fukuoka 812-8581 \\ Japan
}

(E-mail:kaji@math.kyushu-u.ac.jp,mkaneko@math.kyushu-u.ac.jp, tudateru@math.kyushu-u.ac.jp)

\author{
Atsushi Nobe \\ Graduate School of Engineering Science \\ Osaka University \\ 1-3 Machikaneyama-cho \\ Toyonaka \\ Osaka 560-8531 \\ Japan \\ (E-mail:nobe@sigmath.es.osaka-u.ac.jp)
}

